\title{
EXISTENCE RESULTS FOR UNILATERAL QUASISTATIC CONTACT PROBLEMS WITH FRICTION AND ADHESION
}

\author{
Marius $\mathrm{COCU}^{1}$ AND RÉmi RocCA ${ }^{2}$
}

\begin{abstract}
We consider a two dimensional elastic body submitted to unilateral contact conditions, local friction and adhesion on a part of his boundary. After discretizing the variational formulation with respect to time we use a smoothing technique to approximate the friction term by an auxiliary problem. A shifting technique enables us to obtain the existence of incremental solutions with bounds independent of the regularization parameter. We finally obtain the existence of a quasistatic solution by passing to the limit with respect to time.
\end{abstract}

Résumé. Nous considérons un corps élastique bidimensionnel soumis à des conditions de contact unilatéral avec frottement et adhésion sur une partie de sa frontière. Après avoir discrétisé la formulation variationnelle par rapport au temps, nous régularisons le terme de frottement dans un problème auxiliaire. Une technique de translation nous permet d'obtenir l'existence de solutions incrémentales bornées indépendamment du paramètre de régularisation. Nous obtenons finalement l'existence d'une solution quasi-statique en passant à la limite par rapport au temps.

Mathematics Subject Classification. 35K85, 49J40, 73 T05.

Received: February 29, 2000.

\section{INTRODUCTION}

Contact problems for elastic bodies with adhesion and friction appear in many applications of solids mechanics such as the fiber-matrix interface of composite materials. In the present paper we consider the interface model proposed by Cangémi et al. [1-3]. This model contains an internal variable $\beta$ which represents the continuous transition from a total adhesive state to a pure contact state with friction. Quasistatic contact problems with Signorini's condition and local Coulomb friction law have been recently studied by the authors (see [4-6]) and Andersson [7]. There exists at least one solution to such problems if the friction coefficient is sufficiently small. The aim of this paper is to extend the result when adhesion is taken into account at the interface.

In Section 1, we present the mechanical problem and we give a variational formulation $P_{2}$ written as an implicit evolution inequality coupled with a differential equation which represents the evolution of the intensity of adhesion. The body is perfectly bonded to the rigid foundation when $\beta=1$ and there is no more adhesion for $\beta=0$. A time discretization is adopted by using a backward scheme for the implicit inequality and the

\footnotetext{
Keywords and phrases. Friction, adhesion, local Coulomb law, unilateral contact, quasistatic problem.

1 Université de Provence et Laboratoire de mécanique et d'acoustique, CNRS, 31 chemin J. Aiguier, 13402 Marseille Cedex 20, France. e-mail: cocu@lma.cnrs-mrs.fr

2 Laboratoire de mécanique et d'acoustique, CNRS, 31 chemin J. Aiguier, 13402 Marseille Cedex 20, France.

e-mail: rocca@lma.cnrs-mrs.fr
} 
differential equation. We consider a regularized problem associated to the previous one and we present a suitable equivalent problem which allows us to obtain the existence of a solution.

In Section 2, the regularized problem is solved by using a fixed point argument in an auxiliary problem, where the threshold of sliding is given (Tresca's problem). The proof uses a shifting technique and a local straightening of the boundary. By passing to the limit with respect to the regularization parameter, we obtain the existence of a solution to the non regularized incremental problem.

In the last section, we obtain estimates for the difference of two consecutive incremental solutions and we construct suitable sequences of functions for the displacement, the velocity fields and the intensity of adhesion respectively. This enables us to obtain the existence of a solution for the variational formulation of the problem.

\section{Classical and variational formulations}

We consider an elastic body which occupies an open bounded connected subset $\Omega$ of $\mathbb{R}^{2}$ and we denote his boundary by $\partial \Omega$. Let $\Gamma_{1}, \Gamma_{2}$ and $\Gamma_{3}$ three open disjoint parts of $\partial \Omega$ such that $\partial \Omega=\bar{\Gamma}_{1} \cup \bar{\Gamma}_{2} \cup \bar{\Gamma}_{3}$ and $\operatorname{mes}\left(\Gamma_{1}\right)>0$. We denote by $\mathbf{u}=\left(u_{1}, u_{2}\right)$, the displacement field, $\boldsymbol{\epsilon}=\left(\epsilon_{i j}(\mathbf{u})\right)=\frac{1}{2}\left(u_{i, j}+u_{j, i}\right)$, the strain tensor, $\boldsymbol{\sigma}=\left(\sigma_{i j}(\mathbf{u})\right)=\left(a_{i j k l} \epsilon_{k l}(\mathbf{u})\right)$, the stress tensor with the usual summation convention where $i, j, k, l=1,2$. We adopt the following notations for the normal and tangential components of the displacement vector and stress vector $u_{N}=u_{i} n_{i}, \mathbf{u}_{T}=\mathbf{u}-u_{N} \mathbf{n}, \sigma_{N}=\sigma_{i j} n_{i} n_{j},\left(\sigma_{T}\right)_{i}=\sigma_{i j} n_{j}-\sigma_{N} n_{i}$ where $\mathbf{n}=\left(n_{i}\right)$ is the outward unit normal vector to $\partial \Omega$.

We suppose that decohesion occurs on $\Gamma_{3}$ that is the intensity of adhesion is strictly decreasing when the threshold $\mathcal{E}_{a}$ is reached. The parameters $C_{N}$ and $C_{T}>0$ represent the normal and tangential stiffness of the interface when the adhesion is complete that is $\beta=1$. Let us denote by $\phi$ and $\psi$ the densities of the body forces and tractions respectively. The initial displacement of the body is denoted by $\mathbf{u}^{0}$ in $\Omega$ and a displacement $\mathbf{U}=\mathbf{0}$ is prescribed on $\Gamma_{1}$.

Therefore the classical problem is as follows.

Problem $P_{1}:$ Find $\left.\mathbf{u}=\mathbf{u}(\mathbf{x}, t): \Omega \times\right] 0, T\left[\rightarrow \mathbb{R}^{2}, \beta=\beta(\mathbf{x}, t): \Gamma_{3} \times\right] 0, T[\rightarrow[0,1]$ such that

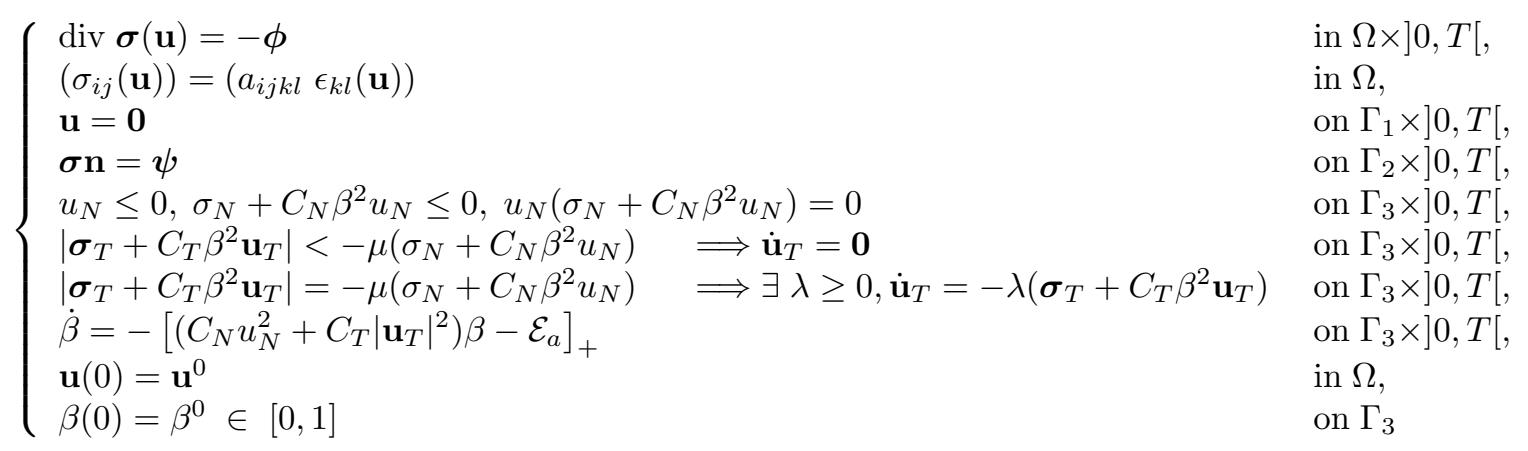

where $\mu$ denotes the friction coefficient and $[s]_{+}=\max (0, s) \forall s \in \mathbb{R}$. We suppose that $a_{i j k l} \in L^{\infty}(\Omega), 1 \leq i, j, k, l \leq 2$, with the usual conditions of symmetry and ellipticity that is

$$
\begin{aligned}
& a_{i j k l}=a_{j i k l}=a_{k l i j}, 1 \leq i, j, k, l \leq 2, \\
& \exists m_{0}>0 \text { such that } \forall \zeta=\left(\zeta_{i j}\right) \in \mathbb{R}^{4}, \text { with } \zeta_{i j}=\zeta_{j i}, 1 \leq i, j \leq 2, a_{i j k l} \zeta_{i j} \zeta_{k l} \geq m_{0}|\zeta|^{2}
\end{aligned}
$$

We require also that $a_{i j k l}$ are $C^{0, \alpha}, 1 \leq i, j, k, l \leq 2,0<\alpha<\frac{1}{2}$ in a neighbourhood of $\Gamma_{3}$. We assume that $\mu \in C^{1}\left(\Gamma_{3}\right)$ with compact support in $\Gamma_{3}$ and the parameters $C_{N}, C_{T}$ belong to $L^{\infty}\left(\Gamma_{3}\right)$. 
The trace mapping will be denoted by $\gamma: H^{1}(\Omega) \rightarrow H^{\frac{1}{2}}(\partial \Omega)$ with norm $\|\gamma\|_{0}$ and the linear bounded extension mapping by $\tilde{\gamma}: H^{\frac{1}{2}}(\partial \Omega) \rightarrow H^{1}(\Omega)$ with norm $\|\tilde{\gamma}\|_{1}$. The norm on $H^{\frac{1}{2}}(\partial \Omega)$ is the following (see for example [12]):

$$
\|w\|_{H^{\frac{1}{2}}(\partial \Omega)}=\|w\|_{L^{2}(\partial \Omega)}+\int_{\partial \Omega} \int_{\partial \Omega} \frac{(w(y)-w(x))^{2}}{|x-y|^{2}} \mathrm{~d} x \mathrm{~d} y .
$$

We still denote by $\gamma$ the following trace mapping $\gamma: H^{1}(\Omega) \rightarrow L^{q}(\partial \Omega)$ which is compact for $1 \leq q<\infty$, (see e.g. [11]). Its norm will be denoted by $\|\gamma\|_{q}$.

We shall omit from now on the notations $\gamma$ and $\tilde{\gamma}$ in order to simplify the notation and we define $\mathrm{V}$ and respectively $\mathrm{K}$ by

$$
V=\left\{\mathbf{v} \in\left[H^{1}(\Omega)\right]^{2}, \mathbf{v}=\mathbf{0} \text { a.e. on } \Gamma_{1}\right\}, K=\left\{\mathbf{v} \in V ; v_{N} \leq 0 \text { a.e. on } \Gamma_{3}\right\} .
$$

Let us equally define $H^{\frac{1}{2}}\left(\Gamma_{3}\right)$ by $H^{\frac{1}{2}}\left(\Gamma_{3}\right)=\left\{w \in H^{\frac{1}{2}}(\partial \Omega) ; w=0\right.$ on $\left.\partial \Omega \backslash \Gamma_{3}\right\}$ equipped with the norm of $H^{\frac{1}{2}}(\partial \Omega)$.

$$
\begin{aligned}
& \langle,\rangle \text { shall denote the duality pairing on } H^{\frac{1}{2}}\left(\Gamma_{3}\right), H^{-\frac{1}{2}}\left(\Gamma_{3}\right), \\
& {[,] \text { " } " \text { " the duality pairing on } H^{\frac{1}{2}}(\partial \Omega), H^{-\frac{1}{2}}(\partial \Omega) \text { and }} \\
& {[,]_{2} \prime \prime \prime \text { the duality pairing on }\left[H^{\frac{1}{2}}(\partial \Omega)\right]^{2},\left[H^{-\frac{1}{2}}(\partial \Omega)\right]^{2} .}
\end{aligned}
$$

We suppose that $\phi \in W^{1,2}\left(0, T ;\left[L^{2}(\Omega)\right]^{2}\right)$ and $\boldsymbol{\psi} \in W^{1,2}\left(0, T ;\left[H^{-\frac{1}{2}}(\partial \Omega)\right]^{2}\right)$ such that there exists an open subset denoted by $\Gamma_{2}^{0}$ with $\operatorname{supp}(\boldsymbol{\psi}(t)) \subset \Gamma_{2}^{0} \subset \bar{\Gamma}_{2}^{0} \subset \Gamma_{2}, \forall t \in[0, T]$.

We define the normal component of the stress vector $\boldsymbol{\sigma n}$ on $\partial \Omega$ at time $\mathrm{t}$ as follows. Let $\mathbf{u} \in\left[H^{1}(\Omega)\right]^{2}$ such that $\operatorname{div} \boldsymbol{\sigma}(\mathbf{u})=-\boldsymbol{\phi}(t)$. Then $\sigma_{N}(\mathbf{u}(t)) \in H^{-\frac{1}{2}}(\partial \Omega)$ is given by

$$
\left[\sigma_{N}(\mathbf{u}), w_{N}\right]=a(\mathbf{u}, \mathbf{w})-(\phi, \mathbf{w})
$$

$\forall \mathbf{w} \in\left[H^{1}(\Omega)\right]^{2}$ such that $\mathbf{w}_{T}=\mathbf{0}$ on $\partial \Omega$

We shall use the notation $\left[s \sigma_{N}(\mathbf{u}), w_{N}\right]=\left[\sigma_{N}(\mathbf{u}), s w_{N}\right], \forall s \in C_{0}^{1}\left(\mathbb{R}^{2}\right)$.

Let us introduce the function $\theta \in C_{0}^{\infty}\left(\mathbb{R}^{2}\right), 0 \leq \theta \leq 1$, such that $\theta=1$ in a neighbourhood of $\bar{\Gamma}_{3}$ and 0 in a neighbourhood of $\overline{\Gamma_{2}^{0}}$. Using Green's formula, we obtain a variational formulation of the problem $P_{1}$ as follows.

Problem $P_{2}$ : Find $(\mathbf{u}, \beta) \in W^{1,2}(0, T ; V) \times W^{1,2}\left(0, T ; L^{\infty}\left(\Gamma_{3}\right)\right)$ such that $\mathbf{u}(0)=\mathbf{u}^{0}, \beta(0)=\beta^{0}$ and for almost all $t \in] 0, T[\mathbf{u}(t) \in K$ and

$$
\begin{aligned}
& a(\mathbf{u}, \mathbf{v}-\dot{\mathbf{u}})+c_{T}(\beta, \mathbf{u}, \mathbf{v}-\dot{\mathbf{u}})+j(\beta, \mathbf{u}, \mathbf{v})-j(\beta, \mathbf{u}, \dot{\mathbf{u}}) \geq(\phi, \mathbf{v}-\dot{\mathbf{u}})+[\boldsymbol{\psi}, \mathbf{v}-\dot{\mathbf{u}}]_{2} \\
& +\left[\sigma_{N}(\mathbf{u}), \theta\left(v_{N}-\dot{u}_{N}\right)\right] \forall v \in V, \\
& {\left[\sigma_{N}(\mathbf{u}), \theta\left(z_{N}-u_{N}\right)\right]+c_{N}(\beta, \mathbf{u}, \mathbf{v}-\mathbf{u}) \geq 0 \forall z \in K,} \\
& \dot{\beta}=-\left[\beta\left(C_{N} u_{N}^{2}+C_{T}\left|\mathbf{u}_{T}\right|^{2}\right)-\mathcal{E}_{a}\right]_{+} \text {a.e. on } \Gamma_{3} \text {, }
\end{aligned}
$$

where $a(\mathbf{u}, \mathbf{v})=\int_{\Omega} a_{i j k l} \epsilon_{i j}(\mathbf{u}) \epsilon_{k l}(\mathbf{v}) \mathrm{d} x, \quad c_{T}(\beta, \mathbf{u}, \mathbf{v})=\int_{\Gamma_{3}} C_{T} \beta^{2} \mathbf{u}_{T} \cdot \mathbf{v}_{T} \mathrm{~d} s, \quad c_{N}(\beta, \mathbf{u}, \mathbf{v})=\int_{\Gamma_{3}} C_{N} \beta^{2} u_{N} v_{N} \mathrm{~d} s$ and $j(\beta, \mathbf{u}, \mathbf{v})=-\left\langle\sigma_{N}(\mathbf{u})+C_{N} \beta^{2} u_{N}, \mu \mid \mathbf{v}_{T}\right\rangle$.

The bilinear form a $(.,$.$) is continuous and coercive by Korn's inequality, as mes \left(\Gamma_{1}\right)>0$, that is a $(.,$.$) satisfies:$

$$
\begin{aligned}
& \exists M>0,|a(\mathbf{u}, \mathbf{v})| \leq M\|\mathbf{u}\|_{\left[H^{1}(\Omega)\right]^{2}}\|\mathbf{v}\|_{\left[H^{1}(\Omega)\right]^{2}}, \quad \forall \mathbf{u}, \mathbf{v} \in\left[H^{1}(\Omega)\right]^{2}, \\
& \exists m>0, a(\mathbf{v}, \mathbf{v}) \geq m\|\mathbf{v}\|_{\left[H^{1}(\Omega)\right]^{2}}^{2}, \quad \forall \mathbf{v} \in V .
\end{aligned}
$$


In order to solve Problem $P_{2}$, we adopt the following time discretization. For all $n \in \mathbb{N}^{*}$, we set $\Delta t=T / n$ and $\phi^{i}=\phi(i \Delta t), \boldsymbol{\psi}^{i}=\boldsymbol{\psi}(i \Delta t)$ for $i=0, \ldots, n$. We assume that the initial displacement $\mathbf{u}^{0}$ belongs to $\mathrm{K}$ and satisfies the following compatibility condition:

$$
a\left(\mathbf{u}^{0}, \mathbf{v}-\mathbf{u}^{0}\right)+c\left(\beta^{0}, \mathbf{u}^{0}, \mathbf{v}-\mathbf{u}^{0}\right)+j\left(\beta^{0}, \mathbf{u}^{0}, \mathbf{v}-\mathbf{u}^{0}\right) \geq\left(\phi^{0}, \mathbf{v}-\mathbf{u}^{0}\right)+\left[\boldsymbol{\psi}^{0}, \mathbf{v}-\mathbf{u}^{0}\right]_{2}, \quad \forall \mathbf{v} \in K
$$

where $c=c_{T}+c_{N}$. We use an implicit scheme which gives the following incremental problem for $i=0, \ldots, n-1$.

Problem $P^{i, n}:$ For $\mathbf{u}^{i} \in K, \beta^{i} \in L^{\infty}\left(\Gamma_{3}\right)$, find $\mathbf{u}^{i+1} \in K, \beta^{i+1} \in L^{\infty}\left(\Gamma_{3}\right)$ such that

$$
\begin{array}{cr}
a\left(\mathbf{u}^{i+1}, \mathbf{v}-\mathbf{u}^{i+1}\right)+c_{T}\left(\beta^{i+1}, \mathbf{u}^{i+1}, \mathbf{v}-\mathbf{u}^{i+1}\right)+j\left(\beta^{i+1}, \mathbf{u}^{i+1}, \mathbf{v}-\mathbf{u}^{i}\right)-j\left(\beta^{i+1}, \mathbf{u}^{i+1}, \Delta \mathbf{u}^{i}\right) \geq & \\
\left(\phi^{i+1}, \mathbf{v}-\mathbf{u}^{i+1}\right)+\left[\boldsymbol{\psi}^{i+1}, \mathbf{v}-\mathbf{u}^{i+1}\right]_{2}+\left[\sigma_{N}\left(\mathbf{u}^{i+1}\right), \theta\left(v_{N}-u_{N}^{i+1}\right)\right] & \forall \mathbf{v} \in V, \\
{\left[\sigma_{N}\left(\mathbf{u}^{i+1}\right), \theta\left(z_{N}-u_{N}^{i+1}\right)\right]+c_{N}\left(\beta^{i+1}, \mathbf{u}^{i+1}, \mathbf{z}-\mathbf{u}^{i+1}\right) \geq 0} & \forall \mathbf{z} \in K, \\
\frac{\beta^{i+1}-\beta^{i}}{\Delta t}=-\left[\beta^{i+1}\left(C_{N}\left(u_{N}^{i+1}\right)^{2}+C_{T}\left|\mathbf{u}_{T}^{i+1}\right|^{2}\right)-\mathcal{E}_{a}\right]_{+} & \text {a.e. on } \Gamma_{3},(8
\end{array}
$$

where $\Delta \mathbf{u}^{i}=\mathbf{u}^{i+1}-\mathbf{u}^{i}$. In order to solve the Problem $P^{i, n}$, we consider the following regularized problem.

Problem $P_{\nu}^{i, n}:$ For $\mathbf{u}^{i} \in K, \beta^{i} \in L^{\infty}\left(\Gamma_{3}\right)$ find $\mathbf{u}_{\nu}^{i+1} \in K, \beta_{\nu}^{i+1} \in L^{\infty}\left(\Gamma_{3}\right)$ such that

$$
\begin{array}{cc}
a\left(\mathbf{u}_{\nu}^{i+1}, \mathbf{v}-\mathbf{u}_{\nu}^{i+1}\right)+c_{T}\left(\beta_{\nu}^{i+1}, \mathbf{u}_{\nu}^{i+1}, \mathbf{v}-\mathbf{u}_{\nu}^{i+1}\right)+j_{\nu}\left(\beta_{\nu}^{i+1}, \mathbf{u}_{\nu}^{i+1}, \mathbf{v}-\mathbf{u}^{i}\right)-j_{\nu}\left(\beta_{\nu}^{i+1}, \mathbf{u}_{\nu}^{i+1}, \mathbf{u}_{\nu}^{i+1}-\mathbf{u}^{i}\right) \geq \\
\left(\phi^{i+1}, \mathbf{v}-\mathbf{u}_{\nu}^{i+1}\right)+\left[\boldsymbol{\psi}^{i+1}, \mathbf{v}-\mathbf{u}_{\nu}^{i+1}\right]_{2}+\left[\sigma_{N}\left(\mathbf{u}_{\nu}^{i+1}\right), \theta\left(v_{N}-u_{\nu N}^{i+1}\right)\right] & \forall \mathbf{v} \in V, \\
{\left[\sigma_{N}\left(\mathbf{u}_{\nu}^{i+1}\right), \theta\left(z_{N}-u_{\nu N}^{i+1}\right)\right]+c_{N}\left(\beta_{\nu}^{i+1}, \mathbf{u}_{\nu}^{i+1}, \mathbf{z}-\mathbf{u}_{\nu}^{i+1}\right) \geq 0} & \forall \mathbf{z} \in K, \\
\frac{\beta_{\nu}^{i+1}-\beta^{i}}{\Delta t}=-\left[\beta_{\nu}^{i+1}\left(C_{N}\left(u_{\nu N}^{i+1}\right)^{2}+C_{T}\left|\mathbf{u}_{\nu T}^{i+1}\right|^{2}\right)-\mathcal{E}_{a}\right]_{+} & \text {a.e. on } \Gamma_{3},
\end{array}
$$

where $j_{\nu}(\beta, \mathbf{u}, \mathbf{v})=-\left\langle\sigma_{N}(\mathbf{u})+C_{N} \beta^{2} u_{N}, \mu \eta_{\nu}\left(\left|\mathbf{v}_{T}\right|\right)\right\rangle$ and the functions $\eta_{\nu}$ with $\nu>0$ have the following properties:

$$
\begin{aligned}
& \eta_{\nu}:\left[0, \infty\left[\rightarrow \left[0, \infty\left[, \eta_{\nu} \in C^{1}\left(\left[0, \infty[), \eta_{\nu}\right.\right. \text { is convex, }\right.\right.\right.\right. \\
& 0 \leq \eta_{\nu}^{\prime} \leq 1, \eta_{\nu}(0)=\eta_{\nu}^{\prime}(0)=0, \\
& \forall s \in\left[0,+\infty\left[, 0 \leq s-\eta_{\nu}(s) \leq \nu .\right.\right.
\end{aligned}
$$

Proposition 1.1. Problem $P_{\nu}^{i, n}$ is equivalent to each of the following problems.

Problem $\bar{P}_{\nu}^{i, n}:$ For $\mathbf{u}^{i} \in K, \beta^{i} \in L^{\infty}\left(\Gamma_{3}\right)$, find $\mathbf{u}_{\nu}^{i+1} \in K, \beta_{\nu}^{i+1} \in L^{\infty}\left(\Gamma_{3}\right)$ such that

$$
\begin{array}{cl}
a\left(\mathbf{u}_{\nu}^{i+1}, \mathbf{w}\right)+c_{T}\left(\beta_{\nu}^{i+1}, \mathbf{u}_{\nu}^{i+1}, \mathbf{w}\right)+j_{\nu}^{\prime}\left(\beta_{\nu}^{i+1}, \mathbf{u}_{\nu}^{i+1}, \Delta_{\nu} \mathbf{u}^{i} ; \mathbf{w}\right)= & \\
\left(\phi^{i+1}, \mathbf{w}\right)+\left[\boldsymbol{\psi}^{i+1}, \mathbf{w}\right]_{2}+\left[\sigma_{N}\left(\mathbf{u}_{\nu}^{i+1}\right), \theta w_{N}\right] & \forall \mathbf{w} \in V, \\
{\left[\sigma_{N}\left(\mathbf{u}_{\nu}^{i+1}\right), \theta\left(z_{N}-u_{\nu N}^{i+1}\right)\right]+c_{N}\left(\beta_{\nu}^{i+1}, \mathbf{u}_{\nu}^{i+1}, \mathbf{z}-\mathbf{u}_{\nu}^{i+1}\right) \geq 0} & \forall \mathbf{z} \in K, \\
\frac{\beta_{\nu}^{i+1}-\beta^{i}}{\Delta t}=-\left[\beta_{\nu}^{i+1}\left(C_{N}\left(u_{\nu N}^{i+1}\right)^{2}+C_{T}\left|\mathbf{u}_{\nu T}^{i+1}\right|^{2}\right)-\mathcal{E}_{a}\right]_{+} & \text {a.e. on } \Gamma_{3},
\end{array}
$$

where $j_{\nu}^{\prime}(\beta, \mathbf{u}, \mathbf{v} ; \mathbf{w})=-\left\langle\sigma_{N}(\mathbf{u})+C_{N} \beta^{2} u_{N}, \mu \eta_{\nu}^{\prime}\left(\left|\mathbf{v}_{T}\right|\right) \mathbf{w}_{T} \cdot \mathbf{d}(\mathbf{v})\right\rangle \forall \mathbf{u}, \mathbf{v}, \mathbf{w} \in V, \quad$ is the Gateaux derivative of $j_{\nu}(\beta, \mathbf{u},),. \mathbf{d}(\mathbf{v})=\frac{\mathbf{v}_{T}}{\left|\mathbf{v}_{T}\right|} \quad$ and $\quad \Delta_{\nu} \mathbf{u}^{i}=\mathbf{u}_{\nu}^{i+1}-\mathbf{u}^{i}$ 
Problem $R_{\nu}^{i, n}:$ For $\mathbf{u}^{i} \in K, \beta^{i} \in L^{\infty}\left(\Gamma_{3}\right)$, find $\mathbf{u}_{\nu}^{i+1} \in K, \beta_{\nu}^{i+1} \in L^{\infty}\left(\Gamma_{3}\right)$ such that

$$
\begin{aligned}
& a\left(\mathbf{u}_{\nu}^{i+1}, \mathbf{v}-\mathbf{u}_{\nu}^{i+1}\right)+c\left(\beta_{\nu}^{i+1}, \mathbf{u}_{\nu}^{i+1}, \mathbf{v}-\mathbf{u}_{\nu}^{i+1}\right)+j_{\nu}^{\prime}\left(\beta_{\nu}^{i+1}, \mathbf{u}_{\nu}^{i+1}, \Delta_{\nu} \mathbf{u}^{i} ; \mathbf{v}-\mathbf{u}_{\nu}^{i+1}\right) \geq \\
& \left(\phi^{i+1}, \mathbf{v}-\mathbf{u}_{\nu}^{i+1}\right)+\left[\boldsymbol{\psi}^{i+1}, \mathbf{v}-\mathbf{u}_{\nu}^{i+1}\right]_{2} \quad \forall \mathbf{v} \in K, \\
& \frac{\beta_{\nu}^{i+1}-\beta^{i}}{\Delta t}=-\left[\beta_{\nu}^{i+1}\left(C_{N}\left(u_{\nu N}^{i+1}\right)^{2}+C_{T}\left|\mathbf{u}_{\nu T}^{i+1}\right|^{2}\right)-\mathcal{E}_{a}\right]_{+} \quad \text { a.e. on } \Gamma_{3} \text {. }
\end{aligned}
$$

Proof. By setting $\mathbf{v}=\mathbf{u}_{\nu}^{i+1} \pm \lambda \mathbf{w}, \lambda \neq 0$, in inequality (9), dividing by $\lambda$ and passing to the limit, one obtains relation (14). Conversely, the convexity of $\eta_{\nu}$ implies that

$$
\eta_{\nu}\left(\left|\left(\mathbf{v}-\mathbf{u}^{i}\right)_{T}\right|\right)-\eta_{\nu}\left(\left|\Delta_{\nu} \mathbf{u}_{T}^{i}\right|\right) \geq \eta_{\nu}^{\prime}\left(\left|\Delta_{\nu} \mathbf{u}_{T}^{i}\right|\right)\left(\mathbf{v}-\mathbf{u}_{\nu}^{i+1}\right)_{T} \cdot \mathbf{d}\left(\Delta_{\nu} \mathbf{u}^{i}\right)
$$

By setting $\mathbf{w}=\mathbf{v}-\mathbf{u}_{\nu}^{i+1}$ in (14), one obtains (9). It is clear that inequalities (14) and (15) implies (17). Using Green's formula in relation (17), we obtain (14) and (15).

We will prove the existence of a solution for Problem $R_{\nu}^{i, n}$ with bounds independent of the regularization parameter in order to obtain the existence of a solution for Problem $P^{i, n}$.

\section{Existence of A SOlution for Problem $P^{i, n}$}

\subsection{Preliminary results}

In order to prove the existence of a solution for Problem $R_{\nu}^{i, n}$ we introduce an operator $\Phi_{0}$ and we give estimates which ensure us that $\Phi_{0}$ has a fixed point.

As $\mu$ is $C^{1}\left(\Gamma_{3}\right)$ it follows that the norm of the following mapping

$$
H^{\frac{1}{2}}\left(\Gamma_{3}\right) \ni v \rightarrow \mu v \in H^{\frac{1}{2}}\left(\Gamma_{3}\right)
$$

is bounded by $\|\mu\|_{H^{\frac{1}{2}}\left(\Gamma_{3}\right)}$.

Lemma 2.1. Assume that $\|\mu\|_{H^{\frac{1}{2}\left(\Gamma_{3}\right)}} \leq \frac{m}{\left(M+C_{N}\right)\|\gamma\|_{0}\|\tilde{\gamma}\|_{1}}$ and there exists a solution $\left(\mathbf{u}_{\nu}^{i+1}, \beta_{\nu}^{i+1}\right)$ to Problem $R_{\nu}^{i, n}$. Then there exists a constant $C_{0}$ such that

$$
\left\|\mathbf{u}_{\nu}^{i+1}\right\|_{\left[H^{1}(\Omega)\right]^{2}} \leq C_{0}\left(\left\|\phi^{i+1}\right\|_{\left[L^{2}(\Omega)\right]^{2}}+\left\|\boldsymbol{\psi}^{i+1}\right\|_{\left[H^{-\frac{1}{2}}(\partial \Omega)\right]^{2}}\right)
$$

Proof. From (12) we have $j_{\nu}^{\prime}\left(\beta_{\nu}^{i+1}, \mathbf{u}_{\nu}^{i+1}, \mathbf{u}_{\nu}^{i+1}-\mathbf{u}^{i} ;-\mathbf{u}_{\nu}^{i+1}\right) \leq j\left(\beta_{\nu}^{i+1}, \mathbf{u}_{\nu}^{i+1}, \mathbf{u}_{\nu}^{i+1}\right)$ so that inequality (17) with $\mathbf{v}=\mathbf{0}$ implies that

$$
a\left(\mathbf{u}_{\nu}^{i+1}, \mathbf{u}_{\nu}^{i+1}\right)+c\left(\beta_{\nu}^{i+1}, \mathbf{u}_{\nu}^{i+1}, \mathbf{u}_{\nu}^{i+1}\right) \leq\left(\phi^{i+1}, \mathbf{u}_{\nu}^{i+1}\right)+\left[\boldsymbol{\psi}^{i+1}, \mathbf{u}_{\nu}^{i+1}\right]_{2}+j\left(\beta_{\nu}^{i+1}, \mathbf{u}_{\nu}^{i+1}, \mathbf{u}_{\nu}^{i+1}\right) .
$$

As $\operatorname{mes}\left(\Gamma_{1}\right)>0$, we obtain:

$m\left\|\mathbf{u}_{\nu}^{i+1}\right\|_{\left[H^{1}(\Omega)\right]^{2}} \leq\left\|\phi^{i+1}\right\|_{\left[L^{2}(\Omega)\right]^{2}}+\left\|\psi^{i+1}\right\|_{\left[H^{-\frac{1}{2}}(\partial \Omega)\right]^{2}}+\|\mu\|_{H^{\frac{1}{2}}\left(\Gamma_{3}\right)}\|\tilde{\gamma}\|_{1}\left\|\sigma_{N}\left(\mathbf{u}_{\nu}^{i+1}\right)+C_{N}\left(\beta_{\nu}^{i+1}\right)^{2} u_{N}^{i+1}\right\|_{H^{-\frac{1}{2}}\left(\Gamma_{3}\right)}$.

Relation (1) enables us to obtain that

$$
\left\|\sigma_{N}\left(\mathbf{u}_{\nu}^{i+1}\right)\right\|_{H^{-\frac{1}{2}}\left(\Gamma_{3}\right)} \leq\|\gamma\|_{0}\left(\left\|\phi^{i+1}\right\|_{\left[L^{2}(\Omega)\right]^{2}}+M\left\|\mathbf{u}_{\nu}^{i+1}\right\|_{\left[H^{1}(\Omega)\right]^{2}}\right)
$$

So inequality (19) follows from the two previous estimates. 
Let $C^{*-}=\left\{g \in H^{-\frac{1}{2}}\left(\Gamma_{3}\right) ; g\right.$ is negative on $\left.\Gamma_{3}\right\}$. For every $g \in C^{*-}$ we define the following contact problem with given friction on the contact zone.

Problem $R_{g}^{i, n}$ : For $\mathbf{u}^{i} \in K, \beta^{i} \in L^{\infty}\left(\Gamma_{3}\right)$, find $\mathbf{u}_{g}^{i+1} \in K, \beta_{g}^{i+1} \in L^{\infty}\left(\Gamma_{3}\right)$ such that

$$
\begin{array}{cc}
a\left(\mathbf{u}_{g}^{i+1}, \mathbf{v}-\mathbf{u}_{g}^{i+1}\right)+c\left(\beta_{g}^{i+1}, \mathbf{u}_{g}^{i+1}, \mathbf{v}-\mathbf{u}_{g}^{i+1}\right)-\left\langle\mu g \mathbf{\Lambda}^{i}, \mathbf{v}_{T}-\mathbf{u}_{g T}^{i+1}\right\rangle \geq & \\
\left(\phi^{i+1}, \mathbf{v}-\mathbf{u}_{g}^{i+1}\right)+\left[\boldsymbol{\psi}^{i+1}, \mathbf{v}-\mathbf{u}_{g}^{i+1}\right]_{2} & \forall \mathbf{v} \in K, \\
\frac{\beta_{g}^{i+1}-\beta^{i}}{\Delta t}=-\left[\beta_{g}^{i+1}\left(C_{N}\left(u_{g N}^{i+1}\right)^{2}+C_{T}\left|\mathbf{u}_{g T}^{i+1}\right|^{2}\right)-\mathcal{E}_{a}\right]_{+} & \text {a.e. on } \Gamma_{3},
\end{array}
$$

where we use the notation $\boldsymbol{\Lambda}^{i}=\eta_{\nu}^{\prime}\left(\left|\left(\mathbf{u}_{g}^{i+1}-\mathbf{u}^{i}\right)_{T}\right|\right) \mathbf{d}\left(\mathbf{u}_{g}^{i+1}-\mathbf{u}^{i}\right)$ and $\left\langle\mu g \boldsymbol{\Lambda}^{i}, \mathbf{v}_{T}\right\rangle=\left\langle\mu g, \boldsymbol{\Lambda}^{i} \cdot \mathbf{v}_{T}\right\rangle$.

Lemma 2.2. Assume that there exists a solution $\left(\mathbf{u}_{g}^{i+1}, \beta_{g}^{i+1}\right)$ to Problem $R_{g}^{i, n}$. Then there exists a constant $C_{1}>0$ such that for all $g \in C^{*-}$ the solution of $R_{g}^{i, n}$ satisfies:

$$
\left\|\mathbf{u}_{g}^{i+1}\right\|_{\left[H^{1}(\Omega)\right]^{2}} \leq C_{1}\left(\left\|\mathbf{u}^{i}\right\|_{\left[H^{1}(\Omega)\right]^{2}}+\left\|\phi^{i+1}\right\|_{\left[L^{2}(\Omega)\right]^{2}}+\left\|\boldsymbol{\psi}^{i+1}\right\|_{\left[H^{-\frac{1}{2}}(\partial \Omega)\right]^{2}}\right) .
$$

Proof. By setting $\mathbf{v}=\mathbf{u}^{i}$ in (20), we get

$$
a\left(\mathbf{u}_{g}^{i+1}, \mathbf{u}_{g}^{i+1}-\mathbf{u}^{i}\right)+c\left(\beta_{g}^{i+1}, \mathbf{u}_{g}^{i+1}, \mathbf{u}_{g}^{i+1}-\mathbf{u}^{i}\right) \underbrace{-\left\langle\mu g \boldsymbol{\Lambda}^{i}, \mathbf{u}_{g T}^{i+1}-\mathbf{u}_{T}^{i}\right\rangle}_{\geq 0} \leq\left(\phi^{i+1}, \mathbf{u}_{g}^{i+1}-\mathbf{u}^{i}\right)+\left[\boldsymbol{\psi}^{i+1}, \mathbf{u}_{g}^{i+1}-\mathbf{u}^{i}\right]_{2} .
$$

The coercivity of a(.,.) enables us to conclude.

We are going to prove that there exists a solution to Problem $R_{g}^{i, n}$. We consider the following problem: for $\beta \in L^{\infty}\left(\Gamma_{3}\right), \mathbf{u}^{i} \in K$, find $\mathbf{u} \in K$ such that

$$
a(\mathbf{u}, \mathbf{v}-\mathbf{u})+c(\beta, \mathbf{u}, \mathbf{v}-\mathbf{u})-\left\langle\mu g \boldsymbol{\Lambda}^{i}, \mathbf{v}_{T}-\mathbf{u}_{T}\right\rangle \geq\left(\phi^{i+1}, \mathbf{v}-\mathbf{u}\right)+\left[\boldsymbol{\psi}^{i+1}, \mathbf{v}-\mathbf{u}\right]_{2} \quad \forall \mathbf{v} \in K .
$$

It is obvious that the problem has a unique solution by a classical argument by using the convexity of $\eta_{\nu}$. We consider equally the following problem.

For $\beta^{i} \in L^{\infty}\left(\Gamma_{3}\right), \mathbf{u} \in K$, find $\beta \in L^{\infty}\left(\Gamma_{3}\right)$ such that

$$
\frac{\beta-\beta^{i}}{\Delta t}=-\left[\beta\left(C_{N} u_{N}^{2}+C_{T}\left|\mathbf{u}_{T}\right|^{2}\right)-\mathcal{E}_{a}\right]_{+} \quad \text { a.e. on } \Gamma_{3} .
$$

This problem has a unique solution which is given by

$$
\beta=\left\{\begin{array}{cc}
\beta^{i} & \text { if }\left(C_{N} u_{N}^{2}+C_{T}\left|\mathbf{u}_{T}\right|^{2}\right) \beta^{i}-\mathcal{E}_{a}<0, \\
\frac{\beta^{i}+\mathcal{E}_{a} \Delta t}{1+\Delta t\left(C_{N} u_{N}^{2}+C_{T}\left|\mathbf{u}_{T}\right|^{2}\right)} & \text { if }\left(C_{N} u_{N}^{2}+C_{T}\left|\mathbf{u}_{T}\right|^{2}\right) \beta^{i}-\mathcal{E}_{a} \geq 0 .
\end{array}\right.
$$

Lemma 2.3. Let $\left(\mathbf{u}_{1}, \mathbf{u}_{2}\right) \in K \times K$ and $\beta_{1}, \beta_{2}$ the corresponding solutions of (24). Let $\tilde{\mathbf{u}}_{1}$ and $\tilde{\mathbf{u}}_{2}$ be the corresponding solutions of (23) with $\beta_{1}, \beta_{2}$. Then there exist two constants $C_{2}>0$ and $C_{3}>0$, independent of $\left(\mathbf{u}_{1}, \mathbf{u}_{2}\right)$ such that

$$
\begin{aligned}
& \left|\beta_{1}-\beta_{2}\right| \leq C_{2} \Delta t\left|\mathbf{u}_{1}-\mathbf{u}_{2}\right|\left|\mathbf{u}_{1}+\mathbf{u}_{2}\right| \quad \text { a.e. on } \Gamma_{3}, \\
& \left\|\tilde{\mathbf{u}}_{1}-\tilde{\mathbf{u}}_{2}\right\|_{\left[H^{1}(\Omega)\right]^{2}} \leq C_{3} \Delta t\left\|\mathbf{u}_{1}-\mathbf{u}_{2}\right\|_{\left[H^{1}(\Omega)\right]^{2}} .
\end{aligned}
$$

Thus there exists a unique solution to Problem $R_{g}^{i, n}$ if $\Delta t$ is small enough. 
Proof. The first inequality follows from the representation of $\beta$. Now in the following inequality equivalent to relation $(23)$

$$
a(\mathbf{u}, \mathbf{v}-\mathbf{u})+c(\beta, \mathbf{u}, \mathbf{v}-\mathbf{u})-\left\langle\mu g, \eta_{\nu}\left(\left|\mathbf{v}_{T}-\mathbf{u}_{T}^{i}\right|\right)-\eta_{\nu}\left(\left|\mathbf{u}_{T}-\mathbf{u}_{T}^{i}\right|\right)\right\rangle \geq\left(\phi^{i+1}, \mathbf{v}-\mathbf{u}\right)+\left[\boldsymbol{\psi}^{i+1}, \mathbf{v}-\mathbf{u}\right]_{2} \quad \forall \mathbf{v} \in K
$$

we introduce $\mathbf{v}=\tilde{\mathbf{u}}_{2}$ with $\beta=\beta_{1}$ and $\mathbf{v}=\tilde{\mathbf{u}}_{1}$ with $\beta=\beta_{2}$. Thus one obtains

$$
a\left(\tilde{\mathbf{u}}_{1}-\tilde{\mathbf{u}}_{2}, \tilde{\mathbf{u}}_{1}-\tilde{\mathbf{u}}_{2}\right)+c_{T}\left(\beta_{2}, \tilde{\mathbf{u}}_{1}-\tilde{\mathbf{u}}_{2}, \tilde{\mathbf{u}}_{1}-\tilde{\mathbf{u}}_{2}\right) \leq \int_{\Gamma_{3}} C_{T}\left(\beta_{1}-\beta_{2}\right)\left(\beta_{1}+\beta_{2}\right) \tilde{\mathbf{u}}_{1 T} \cdot\left(\tilde{\mathbf{u}}_{2 T}-\tilde{\mathbf{u}}_{1 T}\right) \mathrm{d} s .
$$

By the coercivity of $\mathrm{a}(.,$.$) , we have$

$$
m\left\|\tilde{\mathbf{u}}_{1}-\tilde{\mathbf{u}}_{2}\right\|_{\left[H^{1}(\Omega)\right]^{2}} \leq 2 C_{T}\left\|\beta_{1}-\beta_{2}\right\|_{L^{2}\left(\Gamma_{3}\right)}\left\|\tilde{\mathbf{u}}_{1 T}\right\|_{L^{4}\left(\Gamma_{3}\right)}
$$

We conclude by the first relation of the lemma and by a contraction argument when $\Delta t$ is sufficiently small.

In the following, we shall write for the sake of simplicity $(\mathbf{u}, \beta)$ instead of $\left(\mathbf{u}_{g}^{i+1}, \beta_{g}^{i+1}\right)$.

Lemma 2.4. Let the mapping $\Phi_{0}$ from $C^{*-}$ to $H^{-\frac{1}{2}}\left(\Gamma_{3}\right)$ defined by $\Phi_{0}(g)=\sigma_{N}(\mathbf{u})+C_{N} \beta^{2} u_{N}$ with $(\mathbf{u}, \beta)$ solution of $R_{g}^{i, n}$. For every $g \in C^{*-}, \Phi_{0}(g)$ belongs to $C^{*-}$ and there exists a constant $C^{\prime}>0$ such that if $\Delta t<m / C^{\prime}$ then $\Phi_{0}$ is $\frac{1}{2}$ Hölderian on $C^{*-}$.

Proof. Let $g_{j} j=1,2$ and $\mathbf{u}_{j}$ the corresponding solutions. By setting $\mathbf{v}=\mathbf{u}_{2}$ in the equivalent inequality to relation (20) with $g=g_{1}$ and $\mathbf{v}=\mathbf{u}_{1}$ in the equivalent inequality to relation (20) with $g=g_{2}$, we get:

$$
\begin{aligned}
a\left(\mathbf{u}_{1}-\mathbf{u}_{2}, \mathbf{u}_{1}-\mathbf{u}_{2}\right) & +c\left(\beta_{1}, \mathbf{u}_{1}-\mathbf{u}_{2}, \mathbf{u}_{1}-\mathbf{u}_{2}\right) \leq-\left\langle\mu\left(g_{1}-g_{2}\right), \eta_{\nu}\left(\left|\mathbf{u}_{2 T}-\mathbf{u}_{T}^{i}\right|\right)-\eta_{\nu}\left(\left|\mathbf{u}_{1 T}-\mathbf{u}_{T}^{i}\right|\right)\right\rangle \\
& +\int_{\Gamma_{3}}\left[C_{T}\left(\beta_{1}-\beta_{2}\right)\left(\beta_{1}+\beta_{2}\right) \mathbf{u}_{1 T} \cdot\left(\mathbf{u}_{1 T}-\mathbf{u}_{2 T}\right)+C_{N}\left(\beta_{1}-\beta_{2}\right)\left(\beta_{1}+\beta_{2}\right) u_{1 N}\left(u_{1 N}-u_{2 N}\right)\right] \mathrm{d} s
\end{aligned}
$$

It follows that:

$$
\left(m-C^{\prime} \Delta t\right)\left\|\mathbf{u}_{1}-\mathbf{u}_{2}\right\|_{\left[H^{1}(\Omega)\right]^{2}}^{2} \leq C_{1}^{\prime}\left\|g_{1}-g_{2}\right\|_{H^{-\frac{1}{2}\left(\Gamma_{3}\right)}}\left[\left\|\mathbf{u}_{1}\right\|_{\left[H^{1}(\Omega)\right]^{2}}+\left\|\mathbf{u}_{2}\right\|_{\left[H^{1}(\Omega)\right]^{2}}+\left\|\mathbf{u}^{i}\right\|_{\left[H^{1}(\Omega)\right]^{2}}\right]
$$

By using (1) we have

$$
\left\langle\sigma_{N}\left(\mathbf{u}_{1}\right)-\sigma_{N}\left(\mathbf{u}_{2}\right), w_{N}\right\rangle=a\left(\mathbf{u}_{1}-\mathbf{u}_{2}, \mathbf{w}\right) \leq C_{2}^{\prime}\left\|\mathbf{u}_{1}-\mathbf{u}_{2}\right\|_{\left[H^{1}(\Omega)\right]^{2}}\left\|w_{N}\right\|_{H^{\frac{1}{2}}(\partial \Omega)}
$$

Ultimately, we obtain:

$$
\left\|\Phi_{0}\left(g_{2}\right)-\Phi_{0}\left(g_{1}\right)\right\|_{H^{-\frac{1}{2}}\left(\Gamma_{3}\right)} \leq C_{3}^{\prime}\left\|g_{2}-g_{1}\right\|_{H^{-\frac{1}{2}}\left(\Gamma_{3}\right)}^{\frac{1}{2}} \forall g_{1}, g_{2} \in C^{*-}
$$

Let $\mathbf{v}=\mathbf{u}+\mathbf{w}, \mathbf{w} \in\left[H^{1}(\Omega)\right]^{2}$ such that $\mathbf{w}_{T}=\mathbf{0}, w_{N}=0$ on $\partial \Omega \backslash \Gamma_{3}$ and $\left.w_{N}\right|_{\Gamma_{3}} \leq 0$. Then we have

$$
\left\langle\sigma_{N}(\mathbf{u})+C_{N} \beta^{2} u_{N}, w_{N}\right\rangle=a(\mathbf{u}, \mathbf{w})+c_{N}(\beta, \mathbf{u}, \mathbf{w})-\left(\phi^{i+1}, \mathbf{w}\right) \geq\left\langle\mu g \mathbf{\Lambda}^{i}, \mathbf{v}_{T}-\mathbf{u}_{T}\right\rangle=0
$$

By the arbitrariness of $w_{N}$, we deduce that $\Phi_{0}(g) \in C^{*-}$. 


\subsection{Existence of a solution for Problem $\boldsymbol{R}_{\boldsymbol{\nu}}^{i, n}$}

\subsubsection{Case of the strip}

We consider in this part that $\left.\Omega=\Omega_{s}=\mathbb{R} \times\right] 0, r[, r \in] 0,+\infty\left[, \Gamma_{1}=\mathbb{R} \times\{0\}, \Gamma_{2}=\emptyset, \Gamma_{3}=\mathbb{R} \times\{r\}\right.$ and we equally assume that the coefficients $a_{i j k l}$ are $C^{0, \alpha}$ smooth in $\Omega_{s}$.

We define the space $H^{1, \alpha}\left(\Omega_{s}\right)=\left\{w \in H^{1}\left(\Omega_{s}\right) ;\|w\|_{H^{1, \alpha}\left(\Omega_{s}\right)}<+\infty\right\}$, for $0<\alpha<1$, where

$$
\|w\|_{H^{1, \alpha}\left(\Omega_{s}\right)}^{2}=\|w\|_{H^{1}\left(\Omega_{s}\right)}^{2}+\int_{\mathbb{R}} \int_{\Omega_{s}} \frac{1}{|h|^{1+2 \alpha}} \sum_{i=1}^{2}\left[\left(\frac{\partial w}{\partial x_{i}}\right)_{-h}-\left(\frac{\partial w}{\partial x_{i}}\right)\right]^{2} \mathrm{~d} x \mathrm{~d} h
$$

with $v_{-h}\left(x_{1}, x_{2}\right)=v\left(x_{1}+h, x_{2}\right)$, for $\mathbf{x}=\left(x_{1}, x_{2}\right) \in \mathbb{R}^{2}$ and $h \in \mathbb{R}$.

We consider equally the space $H^{\alpha}(\mathbb{R})=\left\{w \in L^{2}(\mathbb{R}) ;\|w\|_{H^{\alpha}(\mathbb{R})}<+\infty\right\}$ with

$$
\|w\|_{H^{\alpha}(\mathbb{R})}^{2}=\|w\|_{L^{2}(\mathbb{R})}^{2}+\int_{\mathbb{R}} \int_{\mathbb{R}} \frac{\left(w\left(x_{1}+h\right)-w\left(x_{1}\right)\right)^{2}}{|h|^{1+2 \alpha}} \mathrm{d} x^{\prime} \mathrm{d} h .
$$

We consider $c_{0}(\alpha)$ such that $|\zeta|^{2 \alpha} c_{0}(\alpha)=\int_{\mathbb{R}^{1}} \frac{|\exp (i h . \zeta)-1|^{2}}{|h|^{1+2 \alpha}} \mathrm{d} h, \forall \zeta \in \mathbb{R}$ and denoting by $F[w]$ the Fourier transform of $w$, we have that

$$
\|w\|_{H^{\alpha}(\mathbb{R})}^{2}=\int_{\mathbb{R}}|F[w](\zeta)|^{2}\left(1+c_{0}(\alpha)|\zeta|^{2 \alpha}\right) \mathrm{d} \zeta \quad(\text { see }[9])
$$

We define $H^{-\alpha}(\mathbb{R})$ as the dual space of $H^{\alpha}(\mathbb{R})$. Thus its norm satisfies

$$
\|w\|_{H^{-\alpha}(\mathbb{R})}^{2}=\int_{\mathbb{R}}|F[w](\zeta)|^{2}\left(1+c_{0}(-\alpha)|\zeta|^{-2 \alpha}\right)^{-1} \mathrm{~d} \zeta \quad(\text { see e.g. } \quad[10]) .
$$

For u solution of $R_{g}^{i, n}$ with $g \in C^{*-} \cap C^{1}(\mathbb{R})$, we can obtain, by applying a shifting technique as in [9] and [5], that for an arbitrary $\epsilon>0$

$$
\left\|\sigma_{N}(\mathbf{u})\right\|_{H^{-\frac{1}{2}+\alpha}(\mathbb{R})} \leq(1+\epsilon)\left(\int_{\mathbb{R}} \frac{M a\left(\mathbf{u}_{-h}-\mathbf{u}, \mathbf{u}_{-h}-\mathbf{u}\right)}{c_{0}(\alpha) c_{0}\left(\frac{1}{2}-\alpha\right)|h|^{1+2 \alpha}} \mathrm{d} h\right)^{\frac{1}{2}}+k_{1}(\epsilon, \alpha)\left[\left\|\phi^{i+1}\right\|_{\left[L^{2}\left(\Omega_{s}\right)\right]^{2}}+\|\mathbf{u}\|_{\left.\left[H^{1}\left(\Omega_{s}\right)\right]^{2}\right]}\right.
$$

with $k_{1}(\epsilon, \alpha)$ a positive constant depending on $\epsilon$ and $\alpha$. Therefore we have

$$
\begin{aligned}
\left\|\Phi_{0}(g)\right\|_{H^{-\frac{1}{2}+\alpha}(\mathbb{R})} \leq & (1+\epsilon)\left(\int_{\mathbb{R}} \frac{M a\left(\mathbf{u}_{-h}-\mathbf{u}, \mathbf{u}_{-h}-\mathbf{u}\right)}{c_{0}(\alpha) c_{0}\left(\frac{1}{2}-\alpha\right)|h|^{1+2 \alpha}} \mathrm{d} h\right)^{\frac{1}{2}} \\
& +k_{2}(\epsilon, \alpha)\left[\left\|\phi^{i+1}\right\|_{\left[L^{2}\left(\Omega_{s}\right)\right]^{2}}+\|\mathbf{u}\|_{\left[H^{1}\left(\Omega_{s}\right)\right]^{2}}\right] .
\end{aligned}
$$

Lemma 2.5. Let $(\mathbf{u}, \beta)$ be a solution of $R_{g}^{i, n}$ with $g \in C^{*-} \cap C^{1}(\mathbb{R})$. Then for an arbitrary $\epsilon>0$ we have

$$
\begin{aligned}
\left(\int_{\mathbb{R}} \frac{m_{0} a\left(\mathbf{u}_{-h}-\mathbf{u}, \mathbf{u}_{-h}-\mathbf{u}\right)}{2 c_{0}(\alpha) c_{0}\left(\frac{1}{2}-\alpha\right)|h|^{1+2 \alpha}} \mathrm{d} h\right)^{\frac{1}{2} \leq} & (1+\epsilon)\|\mu\|_{L^{\infty}\left(\Gamma_{3}\right)}\|g\|_{H^{-\frac{1}{2}+\alpha}(\mathbb{R})} \\
& +k_{2}(\epsilon, \alpha)\left[\left\|\phi^{i+1}\right\|_{\left[L^{2}(\Omega)\right]^{2}}^{2}+\|\mathbf{u}\|_{\left[H^{1}\left(\Omega_{s}\right)\right]^{2}}^{2}\right] .
\end{aligned}
$$


Proof. If we set $\mathbf{v}=\mathbf{u}_{-h}$ in (20), $\mathbf{v}_{-h}=\mathbf{u}$ in the shifted inequality derived from (20) and if we adopt the notation $\boldsymbol{\Lambda}=\boldsymbol{\Lambda}^{i}$ then we have

$$
\begin{aligned}
a\left(\mathbf{u}_{-h}-\mathbf{u}, \mathbf{u}_{-h}-\mathbf{u}\right) \leq & \left\langle(\mu g \boldsymbol{\Lambda})_{-h}-(\mu g \boldsymbol{\Lambda}), \mathbf{u}_{-h T}-\mathbf{u}_{T}\right\rangle \\
& +\left(\phi_{-h}^{i+1}-\phi^{i+1}, \mathbf{u}_{-h}-\mathbf{u}\right)+\left(a_{-h}-a\right)\left(\mathbf{u}_{-h}, \mathbf{u}-\mathbf{u}_{-h}\right) \\
& +c\left(\beta, \mathbf{u}, \mathbf{u}_{-h}-\mathbf{u}\right)+c_{-h}\left(\beta_{-h}, \mathbf{u}_{-h}, \mathbf{u}-\mathbf{u}_{-h}\right) .
\end{aligned}
$$

Then by using the Cauchy-Schwarz inequality we have the following estimate for $0<\alpha^{\prime} \leq 1-2 \alpha$, as $0<\beta<1$ :

$$
\begin{aligned}
\int_{|h|<\delta} \int_{\mathbb{R}} \beta^{2} \frac{u_{N}\left(u_{-h N}-u_{N}\right)}{|h|^{1-\alpha^{\prime}}} \mathrm{d} x^{\prime} & \leq \int_{|h|<\delta}\left(\int_{\mathbb{R}} \frac{u_{N}^{2}}{|h|^{1-\alpha^{\prime}}} \mathrm{d} x^{\prime}\right)^{\frac{1}{2}}\left(\int_{\mathbb{R}} \frac{\left(u_{N}-u_{-h N}\right)^{2}}{|h|^{1+2 \alpha+\alpha^{\prime}}} \mathrm{d} x^{\prime}\right)^{\frac{1}{2}} \mathrm{~d} h \\
& \leq\left(\int_{|h|<\delta} \int_{\mathbb{R}} \frac{u_{N}^{2}}{|h|^{1-\alpha^{\prime}}} \mathrm{d} x^{\prime} \mathrm{d} h\right)^{\frac{1}{2}}\left(\int_{|h|<\delta} \int_{\mathbb{R}} \frac{\left(u_{N}-u_{-h N}\right)^{2}}{|h|^{1+2 \alpha+\alpha^{\prime}}} \mathrm{d} x^{\prime} \mathrm{d} h\right)^{\frac{1}{2}} .
\end{aligned}
$$

By integrating inequality (27) with respect to $\mathrm{h}$ and using the previous estimate, we get (26) with similar arguments as in [5].

Theorem 2.6. Let $(\mathbf{u}, \beta)$ be a solution of $R_{g}^{i, n}$ with $g \in C^{*-} \cap C^{1}(\mathbb{R})$.

If $\|\mu\|_{H^{\frac{1}{2}}\left(\Gamma_{3}\right)}<\frac{m}{\left(M+C_{N}\right)\|\gamma\|_{0}\|\tilde{\gamma}\|_{1}}$ then we have for an arbitrary $\epsilon>0$

$$
\left\|\Phi_{0}(g)\right\|_{H^{-\frac{1}{2}+\alpha}(\mathbb{R})} \leq(1+\epsilon)\left(2 M / m_{0}\right)^{\frac{1}{2}}\|\mu\|_{L^{\infty}\left(\Gamma_{3}\right)}\|g\|_{H^{-\frac{1}{2}+\alpha}(\mathbb{R})}+k_{4}(\epsilon, \alpha)\left\|\phi^{i+1}\right\|_{\left[L^{2}\left(\Omega_{s}\right)\right]^{2}}
$$

If $\mu$ satisfies also $\|\mu\|_{L^{\infty}\left(\Gamma_{3}\right)}<\sqrt{m_{0} / 2 M}$, then Problem $R_{\nu}^{i, n}$ has at least one solution for $\Omega=\Omega_{s}$.

Proof. Inequalities (19), (25) and (26) imply (28), which is valid for all $g \in C^{*-} \cap H^{-\frac{1}{2}+\alpha}(\mathbb{R})$ because $C^{1}(\mathbb{R})$ is dense in $H^{-\frac{1}{2}+\alpha}(\mathbb{R})$.

If $\|\mu\|_{L^{\infty}\left(\Gamma_{3}\right)}<\left(m_{0} / 2 M\right)^{\frac{1}{2}}$, there exists $\epsilon_{0}>0$ such that $\left(1+\epsilon_{0}\right)\left(2 M / m_{0}\right)^{\frac{1}{2}}\|\mu\|_{L^{\infty}\left(\Gamma_{3}\right)}<1$. Thus by (28) there exists $r_{0}>0$ such that $\Phi_{0}$ maps $C^{*-} \cap B_{r_{0}}$ into itself, where $B_{r_{0}}=\left\{g \in H^{-\frac{1}{2}+\alpha}(\mathbb{R}) ;\|g\|_{H^{-\frac{1}{2}+\alpha}(\mathbb{R})} \leq r_{0}\right\}$.

By using Lemma 2.2, we obtain that $\Phi_{0}$ is weakly continuous. Indeed, let $\left(g_{q}\right)_{q \in \mathbb{N}^{*}}$ such that $g_{q} \rightarrow g$ in $H^{-\frac{1}{2}+\alpha}(\mathbb{R})$. As $\mu$ has a compact support we deduce that $\mu g_{q} \rightarrow \mu g$ in $H^{-\frac{1}{2}}(\mathbb{R})$ so that $\Phi_{0}\left(g_{q}\right) \rightarrow \Phi_{0}(g)$ in $H^{-\frac{1}{2}}(\mathbb{R})$. The density of $H^{\frac{1}{2}}(\mathbb{R})$ into $H^{\frac{1}{2}-\alpha}(\mathbb{R})$ implies that $\Phi_{0}\left(g_{q}\right) \rightarrow \Phi(g)$ in $H^{-\frac{1}{2}+\alpha}(\mathbb{R})$.

Hence, we can apply the second Schauder's fixed point theorem (see [13] p. 452) to the mapping $\Phi_{0}$ and there exists at least one solution to Problem $R_{\nu}^{i, n}$ in $\Omega_{s}$.

\subsubsection{Case of a general domain}

In order to apply a shifting technique, we shall adopt the following notations:

$$
\begin{aligned}
& Q_{\delta, r}=\left\{\mathbf{x}=\left(x_{1}, x_{2}\right) \in \mathbb{R}^{2} ;\left|x_{1}\right|<\delta \text { and }\left|x_{2}\right|<r\right\}, \\
& Q_{\delta, r}^{+}=\left\{\mathbf{x}=\left(x_{1}, x_{2}\right) \in \mathbb{R}^{2} ;\left|x_{1}\right|<\delta \text { and } 0<x_{2}<r\right\} \text {, } \\
& Q_{\delta, r}^{0}=\left\{\mathbf{x}=\left(x_{1}, x_{2}\right) \in \mathbb{R}^{2} ;\left|x_{1}\right|<\delta \text { and } x_{2}=0\right\} .
\end{aligned}
$$

Let $\mathbf{x}_{0} \in \Gamma_{3}$ and $U$ be a neighbourhood of $\mathbf{x}_{0}$ such that the coefficients $a_{i j k l}$ are $C^{0, \beta}$ smooth in $\mathrm{U}$. For $\mathbf{w} \in V$ with $\operatorname{supp}(\mathbf{w}) \subset U$ and such that $\mathbf{w}+\mathbf{u} \in K$ relation $(20)$ becomes for $\mathbf{v}=\mathbf{w}+\mathbf{u}$ with $g \in C^{*-} \cap C^{1}\left(\Gamma_{3}\right)$

$$
\int_{\Omega \cap U} a_{i j k l} \epsilon_{i j}(\mathbf{u}) \epsilon_{k l}(\mathbf{w}) \mathrm{d} x+\int_{\Gamma_{3} \cap U} \beta^{2}\left(C_{T} \mathbf{u}_{T} \cdot \mathbf{w}_{T}+C_{N} u_{N} w_{N}\right) \mathrm{d} s-\int_{\Gamma_{3} \cap U} \mu g \boldsymbol{\Lambda} \cdot \mathbf{w}_{T} \mathrm{~d} s \geq \int_{\Omega \cap U} \boldsymbol{\phi}^{i+1} \cdot \mathbf{w} \mathrm{d} x
$$


As $\Gamma_{3}$ is $C^{1, \beta}$ smooth we have that there exists a local representation of the boundary $\zeta_{\mathbf{x}_{0}} \in C^{1, \beta}(\mathbb{R})$ with $x_{2}=\zeta_{x_{0}}\left(x_{1}\right)$ having the following properties. If we set $U_{\delta, r}\left(\mathbf{x}_{0}\right)=\left\{\left(x_{1}, x_{2}\right) \in \mathbb{R}^{2} ;\left|x_{1}\right|<\delta,\left|x_{2}-\zeta_{\mathbf{x}_{0}}\left(x_{1}\right)\right|<r\right\}$, then the mapping $\mathbf{H}_{\delta, r}: U_{\delta, r}\left(\mathbf{x}_{0}\right) \rightarrow Q_{\delta, r}$ defined by $\left(x_{1}, x_{2}\right) \rightarrow\left(x_{1}, x_{2}-\zeta_{x_{0}}\left(x_{1}\right)\right)$ is such that

$$
\mathbf{H}_{\delta, r}\left(U_{\delta, r}\left(\mathbf{x}_{0}\right) \cap \Omega\right)=Q_{\delta, r}^{+}, \quad \mathbf{H}_{\delta, r}\left(U_{\delta, r}\left(\mathbf{x}_{0}\right) \cap \Gamma_{3}\right)=Q_{\delta, r}^{0} .
$$

For any mapping $f$ defined on $U_{\delta, r}\left(\mathbf{x}_{0}\right)$, we denote by $\bar{f}$ the mapping $\bar{f}=f \circ \mathbf{H}^{-1}$.

Therefore, inequality (29) for $U=U_{\delta, r}\left(\mathbf{x}_{0}\right)$ yields

$$
\bar{a}(\overline{\mathbf{u}}, \overline{\mathbf{w}})+b(\overline{\mathbf{u}}, \overline{\mathbf{w}})+\bar{c}(\bar{\beta}, \overline{\mathbf{u}}, \overline{\mathbf{w}})-\int_{Q_{\delta, r}^{0}} \bar{\mu} \bar{g} \bar{J} \overline{\boldsymbol{\Lambda}} \cdot \overline{\mathbf{w}}_{T} \mathrm{~d} x^{\prime} \geq \int_{Q_{\delta, r}^{+}} \bar{\phi}^{i+1} \cdot \overline{\mathbf{w}} \mathrm{d} x
$$

for all $\overline{\mathbf{w}}$ such that $\mathbf{w}+\mathbf{u} \in K$,

$$
\begin{array}{ll}
\text { where } \quad & \bar{a}(\overline{\mathbf{u}}, \overline{\mathbf{w}})=\int_{Q_{\delta, r}^{+}} \bar{a}_{i j k l} \epsilon_{i j}(\overline{\mathbf{u}}) \epsilon_{k l}(\overline{\mathbf{w}}) \mathrm{d} x \\
& b(\overline{\mathbf{u}}, \overline{\mathbf{w}})=\int_{Q_{\delta, r}^{+}} \bar{a}_{1111}\left(\frac{\partial \bar{u}_{1}}{\partial x_{2}} \frac{\partial \bar{w}_{1}}{\partial x_{2}} \frac{\partial \zeta_{\mathbf{x}_{0}}}{\partial x_{1}}-\frac{\partial \bar{u}_{1}}{\partial x_{2}} \frac{\partial \bar{w}_{1}}{\partial x_{1}}-\frac{\partial \bar{u}_{1}}{\partial x_{1}} \frac{\partial \bar{w}_{1}}{\partial x_{2}}\right) \frac{\partial \zeta_{\mathbf{x}_{0}}}{\partial x_{1}} \mathrm{~d} x, \\
& \bar{c}_{T}(\bar{\beta}, \overline{\mathbf{u}}, \overline{\mathbf{w}})=\int_{Q_{\delta, r}^{0}} C_{T} \bar{\beta}^{2} \overline{\mathbf{u}}_{T} \cdot \overline{\mathbf{v}}_{T} \bar{J} \mathrm{~d} x^{\prime}, \\
& \bar{c}_{N}(\bar{\beta}, \overline{\mathbf{u}}, \overline{\mathbf{w}})=\int_{Q_{\delta, r}^{0}} C_{N} \bar{\beta}^{2} \bar{u}_{N} \bar{v}_{N} \bar{J}_{\mathrm{d} x} x^{\prime} \\
\bar{c}(., ., .)=\bar{c}_{T}(., . . .)+\bar{c}_{N}(., . . .) \quad \text { and } \bar{J}^{2}=1+\left(\frac{\partial \zeta_{x_{0}}}{\partial x_{1}}\right)^{2} .
\end{array}
$$

We shall denote by $\bar{b}_{i j k l}$ the coefficients which satisfy the following relation

$$
b(\overline{\mathbf{u}}, \overline{\mathbf{w}})=\int_{Q_{\delta, r}^{+}} \bar{b}_{i j k l} \frac{\partial \bar{u}_{i}}{\partial x_{j}} \frac{\partial \bar{w}_{k}}{\partial x_{l}} \mathrm{~d} x
$$

By using the $C^{0, \beta}$ property of $\frac{\partial \zeta_{\mathbf{x}_{0}}}{\partial x_{1}}$, we have that

$$
|b(\overline{\mathbf{u}}, \overline{\mathbf{w}})| \leq C_{4}^{\prime} \delta^{\beta}\|\mathbf{u}\|_{\left[H^{1}(\Omega)\right]^{2}}\|\mathbf{w}\|_{\left[H^{1}(\Omega)\right]^{2}} .
$$

We extend any function $f$ defined on $U_{\delta, r}\left(x_{0}\right)$ onto $\left.\mathbb{R} \times\right] 0, r\left[\right.$ by $f(\mathbf{x})=f\left(\mathbf{P}_{\delta}^{2}(\mathbf{x})\right)$, where

$$
\mathbf{P}_{\delta}^{2}(\mathbf{x})= \begin{cases}\left(\rho_{0}, z\right) & \text { if }\left|\rho_{0}\right| \leq \delta \\ (\delta, z) & \text { if }\left|\rho_{0}\right|>\delta\end{cases}
$$

if $\left.\mathbf{x}=\left(\rho_{0}, z\right) \in \mathbb{R} \times\right] 0, r[$.

Lemma 2.7. Let $\rho$ be a function such that $\rho \in C_{0}^{\infty}\left(\mathbb{R}^{2}\right)$ with $0 \leq \rho \leq 1$, and $\operatorname{supp}(\rho) \subset U_{\delta, r}\left(\mathbf{x}_{0}\right)$. Let $(\mathbf{u}, \beta)$ be a solution of $R_{g}^{i, n}$ with $g \in C^{*-} \cap C^{1}\left(\Gamma_{3}\right)$. Then we have

$$
\begin{gathered}
\left(\int_{\mathbb{R}} m_{0} \frac{\bar{a}\left(\Delta_{h}(\bar{\rho} \overline{\mathbf{u}}), \Delta_{h}(\bar{\rho} \overline{\mathbf{u}})\right)+b\left(\Delta_{h}(\bar{\rho} \overline{\mathbf{u}}), \Delta_{h}(\bar{\rho} \overline{\mathbf{u}})\right)}{2 c_{0}(\alpha) c_{0}(1 / 2-\alpha)|h|^{1+2 \alpha}} \mathrm{d} h\right)^{\frac{1}{2}} \leq(1+\epsilon+\epsilon(\delta))\|\mu\|_{L^{\infty}\left(\Gamma_{3}\right)} \\
\|\bar{\rho} \bar{J} \bar{g}\|_{H^{-\frac{1}{2}+\alpha}(\mathbb{R})}+k(\epsilon, \alpha) k_{1}^{\prime}\left(\rho, \delta, M, \mu, \phi^{i+1}, \boldsymbol{\psi}^{i+1}, \mathbf{u}^{i}\right)
\end{gathered}
$$

with $\epsilon(\delta) \rightarrow 0$ when $\delta \rightarrow 0$ and $k_{1}^{\prime}(., .,$.$) a constant which depends on \left\|\mathbf{u}^{i}\right\|_{\left[H^{1}(\Omega)\right]^{2}}$. 
Proof. For $h \in \mathbb{R},|h|<\delta / 4$ we denote by $\overline{\mathbf{L}}_{-h}$ the rotation around $\overline{\mathbf{n}}_{-h} \wedge \overline{\mathbf{n}}$ which transforms $\overline{\mathbf{n}}_{-h}$ into $\overline{\mathbf{n}}$. Therefore $\mathbf{L}_{-h} \mathbf{u}_{-h} \in K$ so that we can set $\overline{\mathbf{w}}=\bar{\rho}^{2}\left(\overline{\mathbf{L}}_{-h} \overline{\mathbf{u}}_{-h}-\overline{\mathbf{u}}\right)$ into inequality (30). We equally set $\overline{\mathbf{w}}_{-h}=\bar{\rho}_{-h}^{2}\left(\overline{\mathbf{L}}_{-h}^{-1} \overline{\mathbf{u}}-\overline{\mathbf{u}}_{-h}\right)$ into the shifted inequality derived from (30). By dropping the bar for the sake of simplicity, we obtain

$$
\begin{aligned}
a\left(\Delta_{h}(\rho \mathbf{u}),\right. & \left.\Delta_{h}(\rho \mathbf{u})\right)+b\left(\Delta_{h}(\rho \mathbf{u}), \Delta_{h}(\rho \mathbf{u})\right) \leq\left(\rho \phi^{i+1}, \rho\left(\mathbf{L}_{-h} \mathbf{u}_{-h}-\mathbf{u}\right)\right) \\
& +\left(\left(\rho \phi^{i+1}\right)_{-h}, \rho_{-h}\left(\mathbf{L}_{-h}^{-1} \mathbf{u}-\mathbf{u}_{-h}\right)\right)+\left(a-a_{-h}\right)\left(\rho_{-h} \mathbf{u}_{-h}, \rho_{-h} \mathbf{u}_{-h}-\rho \mathbf{u}\right) \\
& +d^{\prime}\left(\mathbf{u}, \rho\left(\mathbf{L}_{-h} \mathbf{u}_{-h}-\mathbf{u}\right)\right)+a\left(\rho \mathbf{u}, \rho \mathbf{L}_{-h} \mathbf{u}_{-h}-\rho_{-h} \mathbf{u}_{-h}\right) \\
& +d_{-h}^{\prime}\left(\mathbf{u}_{-h}, \rho_{-h}\left(\mathbf{L}_{-h}^{-1} \mathbf{u}-\mathbf{u}_{-h}\right)\right)+a_{-h}\left((\rho \mathbf{u})_{-h}, \rho_{-h} \mathbf{L}_{-h}^{-1} \mathbf{u}-\rho \mathbf{u}\right) \\
& +d^{\prime \prime}\left(\mathbf{u}, \rho\left(\mathbf{L}_{-h} \mathbf{u}_{-h}-\mathbf{u}\right)\right)+b\left(\rho \mathbf{u}, \rho \mathbf{L}_{-h} \mathbf{u}_{-h}-\rho_{-h} \mathbf{u}_{-h}\right) \\
& +d_{-h}^{\prime \prime}\left(\mathbf{u}_{-h}, \rho_{-h}\left(\mathbf{L}_{-h}^{-1} \mathbf{u}-\mathbf{u}_{-h}\right)\right)+b_{-h}\left((\rho \mathbf{u})_{-h}, \rho_{-h} \mathbf{L}_{-h}^{-1} \mathbf{u}-\rho \mathbf{u}\right) \\
& +\left(b-b_{-h}\right)\left(\rho_{-h} \mathbf{u}_{-h}, \rho_{-h} \mathbf{u}_{-h}-\rho \mathbf{u}\right) \\
& +c\left(\beta, \rho \mathbf{u}, \rho\left(\mathbf{L}_{-h} \mathbf{u}_{-h}-\mathbf{u}_{-h}\right)+c_{-h}\left(\beta_{-h},(\rho \mathbf{u})_{-h}, \rho_{-h}\left(\mathbf{L}_{-h}^{-1} \mathbf{u}-\mathbf{u}\right)\right.\right. \\
& +c\left(\beta, \rho \mathbf{u}, \rho\left(\mathbf{u}_{-h}-\mathbf{u}\right)+c_{-h}\left(\beta_{-h},(\rho \mathbf{u})_{-h}, \rho_{-h}\left(\mathbf{u}-\mathbf{u}_{-h}\right)+A^{\prime}\right.\right.
\end{aligned}
$$

where

$$
d^{\prime}(\mathbf{u}, \mathbf{v})=a(\mathbf{u}, \rho \mathbf{v})-a(\rho \mathbf{u}, v), d^{\prime \prime}(\mathbf{u}, \mathbf{v})=b(\mathbf{u}, \rho \mathbf{v})-b(\rho \mathbf{u}, \mathbf{v})
$$

and

$$
\begin{aligned}
A^{\prime}= & -\left\langle\mu g J \boldsymbol{\Lambda},\left(1-\rho^{2}\right) \mathbf{u}_{T}+\rho^{2}\left(\mathbf{L}_{-h} \mathbf{u}_{-h}\right)_{T}-\mathbf{u}_{T}\right\rangle \\
& -\left\langle(\mu g J \boldsymbol{\Lambda})_{-h},\left(\mathbf{u}_{T}\right)_{-h}+\rho_{-h}^{2}\left(\mathbf{L}_{-h}^{-1} \mathbf{u}-\mathbf{u}_{-h}\right)_{T}-\left(\mathbf{u}_{T}\right)_{-h}\right\rangle .
\end{aligned}
$$

Next we have

$$
\begin{aligned}
A^{\prime} \leq & -\left\langle(\mu g J \boldsymbol{\Lambda}) \rho^{2},\left(\mathbf{L}_{-h} \mathbf{u}_{-h}\right)_{T}-\mathbf{u}_{T-h}\right\rangle-\left\langle(\mu g J \boldsymbol{\Lambda})_{-h} \rho_{-h}^{2},\left(\mathbf{L}_{-h}^{-1} \mathbf{u}\right)_{T}-\mathbf{u}_{T}\right\rangle \\
& -\left\langle(\mu g J \boldsymbol{\Lambda}),\left(1-\rho^{2}\right) \mathbf{u}_{T}+\rho^{2}\left(\mathbf{u}_{T}\right)_{-h}-\mathbf{u}_{T}\right\rangle \\
& -\left\langle(\mu g J \boldsymbol{\Lambda})_{-h},\left(1-\rho_{-h}^{2}\right)\left(\mathbf{u}_{T}\right)_{-h}+\rho_{-h}^{2} \mathbf{u}_{T}-\mathbf{u}_{T}\right\rangle
\end{aligned}
$$

Therefore we obtain

$$
\begin{aligned}
A^{\prime} \leq & -\left\langle(\mu g J \boldsymbol{\Lambda}) \rho^{2},\left(\mathbf{L}_{-h} \mathbf{u}_{-h}\right)_{T}-\mathbf{u}_{T-h}\right\rangle-\left\langle(\mu g J \boldsymbol{\Lambda})_{-h} \rho_{-h}^{2},\left(\mathbf{L}_{-h}^{-1} \mathbf{u}\right)_{T}-\mathbf{u}_{T}\right\rangle \\
& -\left\langle(\mu g \boldsymbol{\Lambda} \rho)_{-h},\left(\rho_{-h}-\rho\right) \mathbf{u}_{T}\right\rangle-\left\langle(\mu g \boldsymbol{\Lambda} \rho),\left(\rho_{-h}-\rho\right)\left(\mathbf{u}_{T}\right)_{-h}\right\rangle \\
& +\left\langle(\mu g \boldsymbol{\Lambda} \rho)_{-h}-(\mu g \boldsymbol{\Lambda} \rho),\left(\rho \mathbf{u}_{T}\right)_{-h}-\rho \mathbf{u}_{T}\right\rangle
\end{aligned}
$$

All the expressions in (32) and (33) can be estimated by the Lipschitz continuity of the data (see [9] for L) excepting the last one in (33). By integrating the inequality (32) with respect to $h$, one obtains:

$$
\begin{array}{r}
\int_{|h|<\delta / 4} \frac{a\left(\Delta_{h}(\rho \mathbf{u}), \Delta_{h}(\rho \mathbf{u})\right)+b\left(\Delta_{h}(\rho \mathbf{u}), \Delta_{h}(\rho \mathbf{u})\right)}{|h|^{1+2 \alpha}} \mathrm{d} h \leq k_{2}^{\prime}\left(\rho, \delta, M, \mu, \phi^{i+1}, \psi^{i+1}, \mathbf{u}^{i}\right) k_{1}(\epsilon, \alpha) \\
+(1+\epsilon) \int_{|h|<\delta / 4} \frac{\left\langle(\mu g J \boldsymbol{\Lambda} \rho)_{-h}-(\mu g J \boldsymbol{\Lambda} \rho),\left(\rho \mathbf{u}_{T}\right)_{-h}-\rho \mathbf{u}_{T}\right\rangle}{|h|^{1+2 \alpha}} \mathrm{d} h
\end{array}
$$


As in the case of pure friction (see [5]), we have the following estimate:

$$
\begin{aligned}
\int_{|h|<\delta / 4} \frac{\left\langle(\mu g J \boldsymbol{\Lambda})_{-h}-(\mu g J \boldsymbol{\Lambda} \rho),\left(\rho \mathbf{u}_{T}\right)_{-h}-\rho \mathbf{u}_{T}\right\rangle}{|\mathbf{h}|^{1+2 \alpha}} \mathrm{d} h \leq(1+\epsilon) c_{0}(\alpha)\left(\frac{c_{0}\left(\frac{1}{2}-\alpha\right)}{c_{0}\left(\frac{1}{2}+\alpha\right)}\right)^{\frac{1}{2}} \\
\times\|\mu \rho g J \boldsymbol{\Lambda}\|_{\left[H^{-\frac{1}{2}+\alpha}(\mathbb{R})\right]^{2}}\left\|\rho \mathbf{u}_{T}\right\|_{\left[H^{\frac{1}{2}+\alpha}(\mathbb{R})\right]^{2}}+k(\epsilon, \alpha)\left\|\rho \mathbf{u}_{T}\right\|_{H^{\frac{1}{2}(\mathbb{R})}}^{2}
\end{aligned}
$$

Using that $\left\|\mathbf{u}_{T}\right\|_{\left[H^{\frac{1}{2}+\alpha}(\mathbb{R})\right]^{2}} \leq(1+\epsilon)\|\mathbf{u}\|_{\left[H^{\frac{1}{2}+\alpha}(\mathbb{R})\right]^{2}}+k_{3}(\epsilon)\|\mathbf{u}\|_{\left[L^{2}(\mathbb{R})\right]^{2}}$ and (34), (35) one obtains (31).

Lemma 2.8. Let $\rho$ be a function having the same properties as in Lemma 2.7. Let $(\mathbf{u}, \beta)$ be a solution of $R_{g}^{i, n}$ with $g \in C^{*-} \cap C^{1}\left(\Gamma_{3}\right)$. Then we have

$$
\begin{aligned}
\left\|\bar{\rho} \bar{J} \bar{\sigma}_{N}(\overline{\mathbf{u}})\right\|_{H^{-\frac{1}{2}+\alpha}(\mathbb{R})} \leq & (1+\epsilon)\left(\int_{\mathbb{R}} M \frac{\bar{a}\left(\Delta_{h} \bar{\rho} \overline{\mathbf{u}}, \Delta_{h} \bar{\rho} \overline{\mathbf{u}}\right)+b\left(\Delta_{h} \bar{\rho} \overline{\mathbf{u}}, \Delta_{h} \bar{\rho} \overline{\mathbf{u}}\right)}{c_{0}(\alpha) c_{0}\left(\frac{1}{2}-\alpha\right)|h|^{1+2 \alpha}} \mathrm{d} h\right)^{\frac{1}{2}} \\
& +k_{2}(\epsilon, \alpha) k_{3}^{\prime}\left(\rho, \delta, M, \mu, \phi^{i+1}, \boldsymbol{\psi}^{i+1}, \mathbf{u}^{i}\right)
\end{aligned}
$$

with $\epsilon=\epsilon(\delta) \rightarrow 0$ when $\delta \rightarrow 0$ and $k_{3}^{\prime}(., .,$.$) a constant which depends on \left\|\mathbf{u}^{i}\right\|_{\left[H^{1}(\Omega)\right]^{2}}$.

Proof. Let $\mathbf{w} \in V$ such that $\operatorname{supp}(\mathbf{w}) \subset U$ and such that $w_{N}=0$ on $\partial \Omega \backslash U$. The local straightening of the boundary and (1) yield

$$
\left\langle\bar{\sigma}_{N}(\overline{\mathbf{u}}), \bar{J} \bar{\rho} \bar{w}_{N}\right\rangle=\bar{a}(\bar{\rho} \overline{\mathbf{u}}, \overline{\mathbf{w}})+b(\bar{\rho} \overline{\mathbf{u}}, \overline{\mathbf{w}})+d^{\prime}(\overline{\mathbf{u}}, \overline{\mathbf{w}})+d^{\prime \prime}(\overline{\mathbf{u}}, \overline{\mathbf{w}})-\left(\bar{\rho} \overline{\boldsymbol{\phi}}^{i+1}, \overline{\mathbf{w}}\right) .
$$

As the coefficients $a_{i j k l}$ are $C^{0, \beta}$ in $U_{\delta, r}\left(\mathbf{x}_{0}\right)$, we have

$$
\begin{aligned}
&\left(b_{-h}-b\right)\left(\overline{\mathbf{u}}_{-h}, \overline{\mathbf{w}}\right) \leq C_{1}^{\prime \prime} \chi\left(|h|^{\beta}\right)\|\overline{\mathbf{u}}\|_{\left[H^{1}\left(\Omega_{s}\right)\right]^{2}}\|\overline{\mathbf{w}}\|_{\left[H^{1}\left(\Omega_{s}\right)\right]^{2}}, \\
&\left(d_{-h}^{\prime}-d^{\prime}\right)\left(\overline{\mathbf{u}}_{-h}, \overline{\mathbf{w}}\right) \leq C_{2}^{\prime \prime} \chi\left(|h|^{\beta}\right)\|\overline{\mathbf{u}}\|_{\left[H^{1}\left(\Omega_{s}\right)\right]^{2}}\|\overline{\mathbf{w}}\|_{\left[H^{1}\left(\Omega_{s}\right)\right]^{2}}, \\
&\left(d_{-h}^{\prime \prime}-d^{\prime \prime}\right)\left(\overline{\mathbf{u}}_{-h}, \mathbf{w}\right) \leq C_{3}^{\prime \prime} \chi\left(|h|^{\beta}\right)\|\overline{\mathbf{u}}\|_{\left[H^{1}\left(\Omega_{s}\right)\right]^{2}}\|\overline{\mathbf{w}}\|_{\left[H^{1}\left(\Omega_{s}\right)\right]^{2}}
\end{aligned}
$$

We conclude by the same method as in the case of the strip.

Let $T \in H^{-\frac{1}{2}}\left(\Gamma_{3}\right)$ such that $\operatorname{supp}(\mathrm{T}) \subset \Gamma_{3}$. Let $\left(U_{j}\right)_{j \in I}$ a finite covering of $\Gamma_{3}$ and $\left(\rho_{j}\right)_{j \in I}$ the $C^{\infty}$ partition of unity subordinate to the finite covering. It is well known that the norm |||| $\mid$ defined by

$$
\|T\|^{2}=\sum_{j \in I} \| \bar{\rho}_{j} \bar{T}_{j} \bar{J}_{H^{-\frac{1}{2}+\alpha}(\mathbb{R})}^{2}
$$

is equivalent to the norm $\|T\|_{H^{-\frac{1}{2}+\alpha}\left(\Gamma_{3}\right)}$ (see e.g. [9]).

Let us denote by $\varpi$ a function such that

$$
\varpi \in C_{0}^{\infty}\left(\mathbb{R}^{2}\right), \operatorname{supp}(\varpi) \subset \mathcal{B}\left(\delta_{\mu} / 2\right), \varpi=1 \text { on } \mathcal{B}\left(\delta_{\mu} / 4\right)
$$

where $\delta_{\mu}=\operatorname{dist}\left(\operatorname{supp}(\mu), \partial \Omega \backslash \Gamma_{3}\right)$, and $\mathcal{B}(\alpha)=\left\{\mathbf{x} \in \mathbb{R}^{2} ; \exists \mathbf{y} \in \operatorname{supp}(\mu),|\mathbf{x}-\mathbf{y}|<\alpha\right\}$.

Theorem 2.9. Let $(\mathbf{u}, \beta)$ be a solution of $R_{g}^{i, n}$ with $g \in C^{*-} \cap C^{1}\left(\Gamma_{3}\right)$ and assume that $\|\mu\|_{H^{\frac{1}{2}\left(\Gamma_{3}\right)}}<\frac{m}{\left(M+C_{N}\right)\|\gamma\|_{0}\|\bar{\gamma}\|_{1}}$. Then there exists a finite covering $\left(U_{j}\right)_{j \in I}$ of $\Gamma_{3}$ and a partition of unity $\left(\rho_{j}\right)_{j \in I}$ subordinate to the finite covering such that

$$
\left\|\left|\varpi \Phi_{0}(g)\left\|\left|\leq(1+\epsilon+\epsilon(\delta))\left(m_{0}^{-1} 2 M\right)^{\frac{1}{2}}\|\mu\|_{L^{\infty}\left(\Gamma_{3}\right)}\right|\right\| \varpi g \|\right|+k(\epsilon, \alpha) k_{4}^{\prime}\left(\delta, M, \mu, \phi^{i+1}, \psi^{i+1}\right) .\right.
$$


Thus Problem $P_{\nu}^{i, n}$ has at least one solution if $\|\mu\|_{L^{\infty}\left(\Gamma_{3}\right)}<\sqrt{m_{0} / 2 M}$.

Proof. There exists a finite covering $\left(U_{j}\right)_{j \in I}$ of $\Gamma_{3}$ such that

$$
\begin{aligned}
& \forall j \in I, U_{j}=U_{\delta_{j}, r}\left(\mathbf{x}_{j}\right), \\
& \forall j \in I, U_{j} \cap \operatorname{supp}(\varpi)=\emptyset \text { or } U_{j} \cap\left(\partial \Omega \backslash \Gamma_{3}\right)=\emptyset,
\end{aligned}
$$

with $\delta=\max _{j \in I} \delta_{j}$.

For $j \in I$ such that $U_{j} \cap \operatorname{supp}(\varpi)=\emptyset$ as $\operatorname{supp}\left(\rho_{j}\right) \cap \operatorname{supp}(\mu)=\emptyset$, we have that

$$
\left\|\bar{\varpi} \bar{\rho}_{j} \bar{J}_{j} \bar{\sigma}_{N}(\mathbf{u})\right\|_{H^{-\frac{1}{2}+\alpha}(\mathbb{R})} \leq k_{2}(\epsilon, \alpha) k_{5}^{\prime}\left(\delta_{j}, M, \mu, \phi^{i+1}, \boldsymbol{\psi}^{i+1}, \mathbf{u}^{i}\right) .
$$

For $j \in I$ such that $U_{j} \cap \operatorname{supp}(\varpi)=\emptyset$ we deduce from inequalities (31) and (36) that

$$
\begin{aligned}
\left\|\bar{\varpi} \bar{\rho}_{j} \bar{J}_{j} \bar{\sigma}_{N}(\mathbf{u})\right\|_{H^{-\frac{1}{2}+\alpha}(\mathbb{R})} \leq(1+\epsilon+\epsilon(\delta))\left(m_{0}^{-1} 2 M\right)^{\frac{1}{2}}\|\mu\|_{L^{\infty}\left(\Gamma_{3}\right)}\|\bar{\varpi} \bar{g}\|_{H^{-\frac{1}{2}+\alpha}(\mathbb{R})} & \\
& +k_{2}(\epsilon, \alpha) k_{6}^{\prime}\left(\delta_{j}, M, \mu, \boldsymbol{\phi}^{i+1}, \boldsymbol{\psi}^{i+1}, \mathbf{u}^{i}\right)
\end{aligned}
$$

Therefore from (38), (39) and (19) one obtains (37). The results follows as in the proof of Theorem 2.6.

\subsection{Existence of a solution for Problem $\boldsymbol{P}^{i, n}$}

Theorem 2.10. If $\|\mu\|_{H^{\frac{1}{2}\left(\Gamma_{3}\right)}}<\frac{m}{\left(M+C_{N}\right)\|\gamma\|_{0}\|\tilde{\gamma}\|_{1}}$ and $\|\mu\|_{L^{\infty}\left(\Gamma_{3}\right)}<\sqrt{m_{0} / 2 M}$ then there exists a solution $\left(\mathbf{u}^{i+1}, \beta^{i+1}\right)$ of $P^{i, n}$ such that

$$
\left\|\varpi\left(\sigma_{N}\left(\mathbf{u}^{i+1}\right)+C_{N}\left(\beta^{i+1}\right)^{2} u_{N}^{i+1}\right)\right\|_{H^{-\frac{1}{2}+\alpha}\left(\Gamma_{3}\right)} \leq k_{1}^{\prime \prime}\left(\delta, M, \mu, \phi^{i+1}, \boldsymbol{\psi}^{i+1}\right)
$$

where $k_{1}^{\prime \prime}(., .,$.$) is a constant which depends on \left\|\boldsymbol{\psi}^{i+1}\right\|_{\left[H^{-\frac{1}{2}}(\partial \Omega)\right]^{2}},\left\|\phi^{i+1}\right\|_{\left[L^{2}(\Omega)\right]^{2}}$, $\delta$ and the previous norms of $\mu$. Proof. For each $\nu>0$, there exists $\mathbf{u}_{\nu}^{i+1}$ solution of Problem $P_{\nu}^{i, n}$ where $\mathbf{u}_{\nu}^{i+1}$ satisfies inequality (37) with $g=\sigma_{N}\left(\mathbf{u}_{\nu}^{i+1}\right)+C_{N}\left(\beta_{\nu}^{i+1}\right)^{2} \mathbf{u}_{\nu}^{i+1}$. There exists a subsequence $\left(\mathbf{u}_{\nu_{k}}^{i+1}\right)_{k \in \mathbb{N}^{*}}$ which converges weakly in $\mathrm{V}$ towards a limit denoted by $\mathbf{u}^{i+1}$. We still denote by $\left(\mathbf{u}_{\nu}^{i+1}\right)$ this subsequence.

By a compact imbedding argument, we have that $\left(\beta_{\nu}^{i+1}\right)_{\nu}$ converges strongly towards a limit denoted by $\beta^{i+1}$ and we obtain relation $(7)$.

The weak convergence of $\left(\mathbf{u}_{\nu}^{i+1}\right)$ towards $\mathbf{u}^{i+1}$ implies that

$$
a\left(\mathbf{u}^{i+1}, \mathbf{u}^{i+1}\right) \leq \liminf _{\nu \rightarrow 0^{+}} a\left(\mathbf{u}_{\nu}^{i+1}, \mathbf{u}_{\nu}^{i+1}\right) .
$$

We have equally

$$
c\left(\beta^{i+1}, \mathbf{u}^{i+1}, \mathbf{u}^{i+1}\right)=\lim _{\nu \rightarrow 0^{+}} c\left(\beta_{\nu}^{i+1}, \mathbf{u}_{\nu}^{i+1}, \mathbf{u}_{\nu}^{i+1}\right) .
$$

As $\eta_{\nu}$ satisfies the relation (13) we have $\left|\eta_{\nu}\left(\left|\mathbf{v}_{T}-\mathbf{u}^{i}\right|\right)-\right| \mathbf{v}_{T}-\mathbf{u}_{T}^{i}|| \leq \nu, \quad \forall \nu>0$. So we obtain

$$
\begin{aligned}
\lim _{\nu \rightarrow 0^{+}} j_{\nu}\left(\beta_{\nu}^{i+1}, \mathbf{u}_{\nu}^{i+1}, \mathbf{v}-\mathbf{u}^{i}\right)= & \lim _{\nu \rightarrow 0^{+}}-\left\langle\mu\left(\sigma_{N}\left(\mathbf{u}_{\nu}^{i+1}\right)+C_{N}\left(\beta_{\nu}^{i+1}\right)^{2} u_{\nu N}^{i+1}\right), \eta_{\nu}\left(\left|\mathbf{v}_{T}-\mathbf{u}_{T}^{i}\right|\right)-\left|\mathbf{v}_{T}-\mathbf{u}_{T}^{i}\right|\right\rangle \\
& +\lim _{\nu \rightarrow 0^{+}} j\left(\beta_{\nu}^{i+1}, \mathbf{u}_{\nu}^{i+1}, \mathbf{v}-\mathbf{u}^{i}\right)=j\left(\beta^{i+1}, \mathbf{u}^{i+1}, \mathbf{v}-\mathbf{u}^{i}\right) .
\end{aligned}
$$


Similarly, we have

$$
\liminf _{\nu \rightarrow 0^{+}} j_{\nu}\left(\beta_{\nu}^{i+1}, \mathbf{u}_{\nu}^{i+1}, \mathbf{u}_{\nu}^{i+1}-\mathbf{u}^{i}\right)=\liminf _{\nu \rightarrow 0^{+}} j\left(\beta_{\nu}^{i+1}, \mathbf{u}_{\nu}^{i+1}, \mathbf{u}_{\nu}^{i+1}-\mathbf{u}^{i}\right) .
$$

The strong convergence of $\varpi \sigma_{N}\left(\mathbf{u}_{\nu}^{i+1}\right)$ towards $\varpi \sigma_{N}\left(\mathbf{u}^{i+1}\right)$ in $H^{-\frac{1}{2}}\left(\Gamma_{3}\right)$ due to the compact imbedding of $H^{-\frac{1}{2}+\alpha}\left(\Gamma_{3}\right)$ into $H^{-\frac{1}{2}}\left(\Gamma_{3}\right)$ and the convexity of $j\left(\beta, \mathbf{u}^{i+1},.\right)$ in $H^{-\frac{1}{2}}\left(\Gamma_{3}\right)$ imply that

$$
\liminf _{\nu \rightarrow 0^{+}} j\left(\beta_{\nu}^{i+1}, \mathbf{u}_{\nu}^{i+1}, \mathbf{u}_{\nu}^{i+1}-\mathbf{u}^{i}\right) \geq j\left(\beta^{i+1}, \mathbf{u}^{i+1}, \mathbf{u}^{i+1}-\mathbf{u}^{i}\right) .
$$

By Green's formula it is easy to see that inequalities $(6,7)$ and $(9,10)$ of Problems $P^{i, n}$ and $P_{\nu}^{i, n}$ are respectively equivalent to the following ones:

-find $\mathbf{u}^{i+1} \in K$ such that

$$
\begin{aligned}
a\left(\mathbf{u}^{i+1}, \mathbf{v}-\mathbf{u}^{i+1}\right)+c\left(\beta^{i+1}, \mathbf{u}^{i+1}, \mathbf{v}-\mathbf{u}^{i+1}\right)+j\left(\beta^{i+1}, \mathbf{u}^{i+1}, \mathbf{v}-\mathbf{u}^{i}\right)-j\left(\beta^{i+1}, \mathbf{u}^{i+1}, \Delta \mathbf{u}^{i}\right) \geq \\
\left(\phi^{i+1}, \mathbf{v}-\mathbf{u}^{i+1}\right)+\left[\boldsymbol{\psi}^{i+1}, \mathbf{v}-\mathbf{u}^{i+1}\right]_{2} \quad \forall \mathbf{v} \in K
\end{aligned}
$$

and

-find $\mathbf{u}_{\nu}^{i+1} \in K$ such that

$$
\begin{aligned}
& a\left(\mathbf{u}_{\nu}^{i+1}, \mathbf{v}-\mathbf{u}_{\nu}^{i+1}\right)+c\left(\beta_{\nu}^{i+1}, \mathbf{u}^{i+1}, \mathbf{v}-\mathbf{u}^{i+1}\right)+j_{\nu}\left(\beta_{\nu}^{i+1}, \mathbf{u}_{\nu}^{i+1}, \mathbf{v}-\mathbf{u}^{i}\right)-j_{\nu}\left(\beta_{\nu}^{i+1}, \mathbf{u}_{\nu}^{i+1}, \Delta_{\nu} \mathbf{u}^{i}\right) \geq \\
&\left(\phi^{i+1}, \mathbf{v}-\mathbf{u}_{\nu}^{i+1}\right)+\left[\boldsymbol{\psi}^{i+1}, \mathbf{v}-\mathbf{u}_{\nu}^{i+1}\right]_{2} \quad \forall \mathbf{v} \in K .
\end{aligned}
$$

By using estimates (41) to (45), we can pass to the limit in (47) so that one obtains (46). Relation (40) is obtained by passing to the limit in (37).

\section{Existence of A Solution for Problem $P_{2}$}

Lemma 3.1. If $\|\mu\|_{H^{\frac{1}{2}\left(\Gamma_{3}\right)}}<\frac{m}{\left(M+C_{N}\right)\|\gamma\|_{0}\|\tilde{\gamma}\|_{1}}$ and $\|\mu\|_{L^{\infty}\left(\Gamma_{3}\right)}<\sqrt{m_{0} / 2 M}$ then there exists a positive constant $D_{0}$ such that

$$
\left\|\Delta \mathbf{u}^{i}\right\|_{\left[H^{1}(\Omega)\right]^{2}} \leq D_{0}\left\{\left\|\Delta \phi^{i}\right\|_{\left[L^{2}(\Omega)\right]^{2}}+\left\|\Delta \psi^{i}\right\|_{\left[H^{-\frac{1}{2}}(\Omega)\right]^{2}}+\Delta t\right\}
$$

where $\mathbf{u}^{i}$ and $\mathbf{u}^{i+1}$ are solutions to $P^{i, n}$ and $P^{i-1, n}$.

Proof. By setting $\mathbf{v}=\mathbf{u}^{i}$ in inequality (46), we have

$$
a\left(\mathbf{u}^{i+1}, \Delta \mathbf{u}^{i}\right)+c\left(\beta^{i+1}, \mathbf{u}^{i+1}, \Delta \mathbf{u}^{i}\right)+j\left(\beta^{i+1}, \mathbf{u}^{i+1}, \Delta \mathbf{u}^{i}\right) \leq\left(\phi^{i+1}, \Delta \mathbf{u}^{i}\right)+\left[\boldsymbol{\psi}^{i+1}, \Delta \mathbf{u}^{i}\right]_{2} .
$$

We equally set $\mathbf{v}=\mathbf{u}^{i+1}$ in the inequality (46) corresponding to Problem $P^{i-1, n}$, so that we obtain

$$
a\left(\mathbf{u}^{i}, \Delta \mathbf{u}^{i}\right)+c\left(\beta^{i}, \mathbf{u}^{i}, \Delta \mathbf{u}^{i}\right)+j\left(\beta^{i}, \mathbf{u}^{i}, \mathbf{u}^{i+1}-\mathbf{u}^{i-1}\right)-j\left(\beta^{i}, \mathbf{u}^{i}, \Delta \mathbf{u}^{i-1}\right) \geq\left(\phi^{i}, \Delta \mathbf{u}^{i}\right)+\left[\boldsymbol{\psi}^{i}, \Delta \mathbf{u}^{i}\right]_{2} .
$$

Using that $\left|\mathbf{u}_{T}^{i+1}-\mathbf{u}_{T}^{i-1}\right| \leq\left|\Delta \mathbf{u}_{T}^{i}\right|+\left|\Delta \mathbf{u}_{T}^{i-1}\right|$ and adding the two last inequalities we have

$$
\begin{array}{r}
a\left(\Delta \mathbf{u}^{i}, \Delta \mathbf{u}^{i}\right)+c\left(\beta^{i+1}, \Delta \mathbf{u}^{i}, \Delta \mathbf{u}^{i}\right)+j\left(\beta^{i+1}, \Delta \mathbf{u}^{i}, \Delta \mathbf{u}^{i}\right) \leq\left\langle\mu C_{N}\left[\left(\beta^{i+1}\right)^{2}-\left(\beta^{i}\right)^{2}\right] u_{N}^{i+1},\left|\Delta \mathbf{u}_{T}^{i}\right|\right\rangle \\
+\int_{\Gamma_{3}} C_{T}\left(\beta^{i}-\beta^{i+1}\right)\left(\beta^{i}+\beta^{i+1}\right) \mathbf{u}_{T}^{i}\left(\mathbf{u}_{T}^{i+1}-\mathbf{u}_{T}^{i}\right)+C_{N}\left(\beta^{i}-\beta^{i+1}\right)\left(\beta^{i}+\beta^{i+1}\right) u_{N}^{i}\left(u_{N}^{i+1}-u_{N}^{i}\right) \mathrm{d} s \\
+\left(\Delta \phi^{i}, \Delta \mathbf{u}^{i}\right)+\left[\Delta \psi^{i}, \Delta \mathbf{u}^{i}\right]_{2} .
\end{array}
$$


Therefore we obtain that

$$
\left|j\left(\beta^{i}, \Delta \mathbf{u}^{i}, \Delta \mathbf{u}^{i}\right)\right| \leq\|\mu\|_{H^{\frac{1}{2}}\left(\Gamma_{3}\right)}\|\gamma\|_{0}\left\|\sigma_{N}\left(\Delta \mathbf{u}^{i}\right)+C_{N}\left(\beta^{i}\right)^{2} u_{N}^{i}\right\|_{H^{-\frac{1}{2}}\left(\Gamma_{3}\right)}\left\|\Delta \mathbf{u}^{i}\right\|_{\left[H^{1}(\Omega)\right]^{2}}
$$

Relation (1) allows us to deduce that

$$
\left\|\sigma_{N}\left(\Delta \mathbf{u}^{i}\right)\right\|_{H^{-\frac{1}{2}}\left(\Gamma_{3}\right)} \leq\|\tilde{\gamma}\|_{1}\left(M\left\|\Delta \mathbf{u}^{i}\right\|_{\left[H^{1}(\Omega)\right]^{2}}+\left\|\Delta \phi^{i}\right\|_{\left[L^{2}(\Omega)\right]^{2}}\right)
$$

We have also

$$
\left\|\beta^{i+1}-\beta^{i}\right\|_{L^{2}\left(\Gamma_{3}\right)} \leq D_{2}^{\prime} \Delta t
$$

Hence (48) results from the coercivity of a $(.,$.$) .$

We consider the sequences of functions $\left(\beta_{n}\right)_{n \in \mathbb{N}^{*}},\left(\mathbf{u}_{n}\right)_{n \in \mathbb{N}^{*}},\left(\tilde{\mathbf{u}}_{n}\right)_{n \in \mathbb{N}^{*}},\left(\boldsymbol{\phi}_{n}\right)_{n \in \mathbb{N}^{*}},\left(\boldsymbol{\psi}_{n}\right)_{n \in \mathbb{N}^{*}}$ defined on $[0, T]$ by $\beta_{n}(t)=\beta^{i+1}, \quad \mathbf{u}_{n}(t)=\mathbf{u}^{i+1}, \quad \tilde{\mathbf{u}}_{n}(t)=\mathbf{u}^{i}+\left(t-t_{i}\right)\left(\mathbf{u}^{i+1}-\mathbf{u}^{i}\right) / \Delta t, \quad \phi_{n}(t)=\phi^{i+1}, \quad \boldsymbol{\psi}_{n}(t)=\boldsymbol{\psi}^{i+1}$ for $\left.t \in] t_{i}, t_{i+1}\right]$, with $t_{i}=i . \Delta t, i=0, . ., n-1$ and $\beta_{n}(0)=\beta^{0}, \mathbf{u}_{n}(0)=\tilde{\mathbf{u}}_{n}(0)=\mathbf{u}^{0}, \phi_{n}(0)=\phi^{0}, \boldsymbol{\psi}_{n}(0)=\boldsymbol{\psi}^{0}$.

Lemma 3.2. Let $i=0,1, . ., n-1$ and $0 \leq t_{1}<t_{2} \leq T$. Then there exist positive constants $D_{1}, D_{2}, D_{3}$ such that

$$
\begin{gathered}
\left\|\Delta \mathbf{u}^{i}\right\|_{\left[H^{1}(\Omega)\right]^{2}} \leq D_{1} \int_{t_{i}}^{t_{i+1}}\left(1+\|\dot{\phi}(t)\|_{\left[L^{2}(\Omega)\right]^{2}}+\|\dot{\boldsymbol{\psi}}(t)\|_{\left[H^{-\frac{1}{2}}(\partial \Omega)\right]^{2}}\right) \mathrm{d} t \\
\left\|\Delta \mathbf{u}^{i}\right\|_{\left[H^{1}(\Omega)\right]^{2}}^{2} \leq D_{2} \Delta t \int_{t_{i}}^{t_{i+1}}\left(1+\|\dot{\boldsymbol{\phi}}(t)\|_{\left[L^{2}(\Omega)\right]^{2}}^{2}+\|\dot{\boldsymbol{\psi}}(t)\|_{\left[H^{-\frac{1}{2}}(\partial \Omega)\right]^{2}}^{2}\right) \mathrm{d} t \\
\left\|\mathbf{u}_{n}\left(t_{1}\right)-\mathbf{u}_{n}\left(t_{2}\right)\right\|_{\left[H^{1}(\Omega)\right]^{2}} \leq D_{3} \int_{t_{1}}^{\min \left(t_{2}+\Delta t, T\right)}\left(1+\|\dot{\boldsymbol{\phi}}(t)\|_{\left[L^{2}(\Omega)\right]^{2}}+\|\dot{\boldsymbol{\psi}}(t)\|_{\left[H^{\left.-\frac{1}{2}(\partial \Omega)\right]^{2}}\right.}\right) \mathrm{d} t
\end{gathered}
$$

Proof. Inequality (48) and the absolute continuity of functions $\boldsymbol{\phi}$ and $\boldsymbol{\psi}$ imply relation (49). We obtain estimate (50) by using Cauchy-Schwarz inequality in relation (49). Let $0<i<j<n$ such that $\left.\left.t_{1} \in\right] t_{i}, t_{i+1}\right]$, $\left.\left.t_{2} \in\right] t_{j}, t_{j+1}\right]$

$$
\begin{aligned}
\left\|\mathbf{u}_{n}\left(t_{1}\right)-\mathbf{u}_{n}\left(t_{2}\right)\right\|_{\left[H^{1}(\Omega)\right]^{2}} & \leq \sum_{m=i+1}^{j}\left\|\mathbf{u}^{m+1}-\mathbf{u}^{m}\right\|_{\left[H^{1}(\Omega)\right]^{2}} \\
& \leq D_{3} \sum_{m=i+1}^{j} \int_{t_{m}}^{t_{m+1}}\left(1+\|\dot{\boldsymbol{\phi}}(t)\|_{\left[L^{2}(\Omega)\right]^{2}}+\|\dot{\boldsymbol{\psi}}(t)\|_{\left[H^{-\frac{1}{2}}(\partial \Omega)\right]^{2}}\right) \mathrm{d} t \\
& \leq D_{3} \int_{t_{i}+1}^{t_{j+1}}\left(1+\|\dot{\boldsymbol{\phi}}(t)\|_{\left[L^{2}(\Omega)\right]^{2}}+\|\dot{\boldsymbol{\psi}}(t)\|_{\left[H^{\left.-\frac{1}{2}(\partial \Omega)\right]^{2}}\right.}\right) \mathrm{d} t
\end{aligned}
$$

so that (51) holds.

Lemma 3.3. There exist two subsequences $\left(\mathbf{u}_{n_{k}}\right)_{k \in \mathbb{N}^{*}},\left(\tilde{\mathbf{u}}_{n_{k}}\right)_{k \in \mathbb{N}^{*}}$ and an element $\mathbf{u} \in W^{1,2}(0, T ; V)$ such that for all $t \in[0, T]\left(\mathbf{u}_{n_{k}}(t)\right)_{k \in \mathbb{N}^{*}}$ converges weakly in $V$ towards $\mathbf{u}(t)$ and $\left(\tilde{\mathbf{u}}_{n_{k}}\right)_{k \in \mathbb{N}^{*}}$ converges weakly in $W^{1,2}(0, T ; V)$ towards $\mathbf{u}$. 
Proof. First by (50) we have

$$
\begin{aligned}
\left\|\tilde{\mathbf{u}}_{n}\right\|_{W^{1,2}(0, T ; V)}^{2} & \leq \int_{0}^{T}\left(\left\|\tilde{\mathbf{u}}_{n}(t)\right\|_{\left[H^{1}(\Omega)\right]^{2}}^{2}+\left\|\frac{d}{d t} \tilde{\mathbf{u}}_{n}(t)\right\|_{\left[H^{1}(\Omega)\right]^{2}}^{2}\right) \mathrm{d} t \\
& \leq D_{4} T+\sum_{i=0}^{n-1} \int_{t_{i}}^{t_{i+1}}\left\|\frac{\mathbf{u}^{i+1}-\mathbf{u}^{i}}{\Delta t}\right\|_{\left[H^{1}(\Omega)\right]^{2}}^{2} \mathrm{~d} t \\
& \leq D_{4} T+\sum_{i=0}^{n-1} \int_{t_{i}}^{t_{i+1}} \frac{D_{2}}{\Delta t}\left(\int_{t_{i}}^{t_{i+1}}\left(1+\|\dot{\boldsymbol{\phi}}(\tau)\|_{\left[L^{2}(\Omega)\right]^{2}}^{2}+\|\dot{\boldsymbol{\psi}}(\tau)\|_{\left[H^{\left.-\frac{1}{2}(\partial \Omega)\right]^{2}}\right.}^{2}\right) \mathrm{d} \tau\right) \mathrm{d} t<+\infty
\end{aligned}
$$

Next by using an argument similar as in [8], we obtain the existence of $\left(\mathbf{u}_{n}\right)_{n \in \mathbb{N}^{*}},\left(\tilde{\mathbf{u}}_{n}\right)_{n \in \mathbb{N}^{*}}$ and an element $\mathbf{u} \in W^{1,2}(0, T ; V)$ such that $\left(\mathbf{u}_{n}(t)\right)_{n \in \mathbb{N}^{*}}$ converges weakly towards $\mathbf{u}(t) \forall t \in[0, T]$. Moreover for all $\boldsymbol{\vartheta} \in$ $L^{2}(0, T ; V)$ we have

$$
\left|\left(\tilde{\mathbf{u}}_{n_{k}}-\mathbf{u}_{n_{k}}, \boldsymbol{\vartheta}\right)\right| \leq D_{5} \Delta t\left(\int_{0}^{T}\left(1+\|\dot{\boldsymbol{\phi}}(t)\|_{\left[L^{2}(\Omega)\right]^{2}}^{2}+\|\dot{\boldsymbol{\psi}}(t)\|_{\left[H^{-\frac{1}{2}}(\partial \Omega)\right]^{2}}^{2}\right) \mathrm{d} t\right)^{\frac{1}{2}} \times\left(\int_{0}^{T}\|\boldsymbol{\vartheta}(t)\|_{\left[H^{1}(\Omega)\right]^{2}}^{2} \mathrm{~d} t\right)^{\frac{1}{2}},
$$

so that $\left(\tilde{\mathbf{u}}_{n_{k}}-\mathbf{u}_{n_{k}}, \boldsymbol{\vartheta}\right) \rightarrow 0 \forall t \in[0, T]$. As $\left(\mathbf{u}_{n_{k}}(t)\right)_{k \in \mathbb{N}^{*}}$ converges weakly towards $\mathbf{u}(t), \forall t \in[0, T]$ we finally obtain that $\left(\tilde{\mathbf{u}}_{n_{k}}\right)_{k \in \mathbb{N}^{*}}$ converges weakly in $W^{1,2}(0, T ; V)$ towards $\mathbf{u}$.

From now on, we still denote by $\left(\mathbf{u}_{n}\right)_{n \in \mathbb{N}^{*}},\left(\tilde{\mathbf{u}}_{n}\right)_{n \in \mathbb{N}^{*}},\left(\phi_{n}\right)_{n \in \mathbb{N}^{*}},\left(\boldsymbol{\psi}_{n}\right)_{n \in \mathbb{N}^{*}}$ and $\left(\beta_{n}\right)_{n \in \mathbb{N}^{*}}$ the subsequences $\left(\mathbf{u}_{n_{k}}\right)_{k \in \mathbb{N}^{*}},\left(\tilde{\mathbf{u}}_{n_{k}}\right)_{k \in \mathbb{N}^{*}},\left(\phi_{n_{k}}\right)_{k \in \mathbb{N}^{*}},\left(\boldsymbol{\psi}_{n_{k}}\right)_{k \in \mathbb{N}^{*}}$ and $\left(\beta_{n_{k}}\right)_{k \in \mathbb{N}^{*}}$.

Lemma 3.4. There exists a unique $\beta \in W^{1,2}\left(0, T ; L^{2}\left(\Gamma_{3}\right)\right)$ solution of (4) corresponding to the weak limit $\mathbf{u}$.

Proof. Let $X=\left\{\beta \in C^{0}\left([0, T] ; L^{2}\left(\Gamma_{3}\right)\right) ; \sup _{t \in[0, T]}\left[\exp (-k t)\|\beta(t)\|_{L^{2}\left(\Gamma_{3}\right)}\right]<+\infty\right\} . X$ is a Banach space for the norm $\|\beta\|_{X}=\sup _{t \in[0, T]}\left[\exp (-k t)\|\beta(t)\|_{L^{2}\left(\Gamma_{3}\right)}\right]$. Let $\mathcal{T}: X \rightarrow X$ be the mapping defined by

$$
\mathcal{T} \beta(t)=\beta_{0}-\int_{0}^{t}\left[\left(C_{N} u_{N}^{2}+C_{T}\left|\mathbf{u}_{T}\right|^{2}\right) \beta-\mathcal{E}_{a}\right]_{+} \mathrm{d} \tau
$$

We have

$$
\left|\mathcal{T} \beta_{1}(t)-\mathcal{T} \beta_{2}(t)\right|^{2} \leq D_{1}^{\prime} \int_{0}^{t}\left(C_{N} u_{N}^{2}+C_{T}\left|\mathbf{u}_{T}\right|^{2}\right)^{2}\left(\beta_{1}-\beta_{2}\right)^{2} \mathrm{~d} \tau
$$

As $\mathbf{u}(t)$ belongs to $\left[H^{\frac{1}{2}+\alpha}\left(\Gamma_{3}\right)\right]^{2}$ for all $t \in[0, T], \mathbf{u}(t)$ is continuous on $\Gamma_{3}$. Thus we obtain that

$$
\begin{aligned}
\left\|\mathcal{T} \beta_{1}(t)-\mathcal{T} \beta_{2}(t)\right\|_{L^{2}\left(\Gamma_{3}\right)}^{2} & \leq D_{2}^{\prime}\|\mathbf{u}\|_{L^{\infty}(] 0, T\left[\times \Gamma_{3}\right)}^{4} \int_{0}^{t}\left\|\beta_{1}-\beta_{2}\right\|_{L^{2}\left(\Gamma_{3}\right)}^{2} \mathrm{~d} s \\
& \leq D_{2}^{\prime}\|\mathbf{u}\|_{L^{\infty}(] 0, T\left[\times \Gamma_{3}\right)}^{4}\left\|\beta_{1}-\beta_{2}\right\|_{X}^{2} \frac{\exp (2 k t)}{2 k} .
\end{aligned}
$$

It follows that

$$
\left\|\mathcal{T} \beta_{1}(t)-\mathcal{T} \beta_{2}(t)\right\|_{X} \leq \sqrt{\frac{D_{2}^{\prime}}{2 k}}\|\mathbf{u}\|_{L^{\infty}(] 0, T\left[\times \Gamma_{3}\right)}^{2}\left\|\beta_{1}-\beta_{2}\right\|_{X} .
$$

There exists $\mathrm{k}$ sufficiently large such that $\mathcal{T}$ has a fixed point $\beta$. 
Next we are going to show that $(\mathbf{u}, \beta)$ is a solution of the quasistatic problem. First, we prove that $\left(\beta_{n}\right)_{n \in \mathbb{N}^{*}}$ converges strongly towards $\beta$ in $L^{\infty}\left(0, T ; L^{2}\left(\Gamma_{3}\right)\right)$.

Lemma 3.5. We have the following result: $\lim _{n \rightarrow+\infty} \max _{i=0, ., n}\left\|e_{n}^{i}\right\|_{L^{2}\left(\Gamma_{3}\right)}=0$ where $e_{n}^{i}=\beta\left(t_{i}\right)-\beta^{i}$.

Proof. We introduce the following notation

$$
y(\beta(t), \mathbf{u}(t))=-\left[\left(C_{N} u_{N}^{2}(t)+C_{T}\left|\mathbf{u}_{T}(t)\right|^{2}\right) \beta(t)-\mathcal{E}_{a}\right]_{+} .
$$

The following relation holds

$$
e_{n}^{i+1}=e_{n}^{i}+\Delta t\left[y\left(\beta\left(t_{i+1}\right), \mathbf{u}\left(t_{i+1}\right)\right)-y\left(\beta^{i+1}, \mathbf{u}^{i+1}\right)\right]+\epsilon_{n}^{i}
$$

with $\epsilon_{n}^{i}=\int_{t_{i}}^{t_{i+1}}\left[y(\beta(\tau), \mathbf{u}(\tau))-y\left(\beta\left(t_{i+1}\right), \mathbf{u}\left(t_{i+1}\right)\right)\right] \mathrm{d} \tau$. Therefore we get

$$
\left\|e_{n}^{i+1}\right\|_{L^{2}\left(\Gamma_{3}\right)} \leq\left\|e_{n}^{i}\right\|_{L^{2}\left(\Gamma_{3}\right)}+\Delta t D_{2}^{\prime \prime}\left\|e_{n}^{i+1}\right\|_{L^{2}\left(\Gamma_{3}\right)}+D_{3}^{\prime \prime} \Delta t\left\|\mathbf{u}^{2}\left(t_{i+1}\right)-\left(\mathbf{u}^{i+1}\right)^{2}\right\|_{\left[L^{2}\left(\Gamma_{3}\right)\right]^{2}}+\left\|\epsilon_{n}^{i+1}\right\|_{L^{2}\left(\Gamma_{3}\right)} .
$$

We choose $\Delta t \leq 1 / D_{2}^{\prime \prime}$ and we obtain

$$
\left\|e_{n}^{i+1}\right\|_{L^{2}\left(\Gamma_{3}\right)} \leq \frac{1}{\left(1-D_{2}^{\prime \prime} \Delta t\right)}\left(\left\|e_{n}^{i}\right\|_{L^{2}\left(\Gamma_{3}\right)}+D_{3}^{\prime \prime} \Delta t\left\|\mathbf{u}^{2}\left(t_{i+1}\right)-\left(\mathbf{u}^{i+1}\right)^{2}\right\|_{\left[L^{2}\left(\Gamma_{3}\right)\right]^{2}}+\left\|\epsilon_{n}^{i+1}\right\|_{L^{2}\left(\Gamma_{3}\right)}\right) .
$$

Using the relation $1+x \leq \exp (x), \forall x \in \mathbb{R}$ and $\left\|e_{n}^{0}\right\|_{L^{2}\left(\Gamma_{3}\right)}=0$, we obtain that

$$
\left\|e_{n}^{i}\right\|_{L^{2}\left(\Gamma_{3}\right)} \leq \sum_{k=0}^{i-1} \exp \left[\frac{D_{2}^{\prime \prime}}{1-D_{2}^{\prime \prime} \Delta t}\left(t_{i}-t_{k+1}\right)\right]\left[D_{3}^{\prime \prime} \Delta t\left\|\mathbf{u}^{2}\left(t_{k+1}\right)-\left(\mathbf{u}^{k+1}\right)^{2}\right\|_{\left[L^{2}\left(\Gamma_{3}\right)\right]^{2}}+\left\|\epsilon_{n}^{k+1}\right\|_{L^{2}\left(\Gamma_{3}\right)}\right] .
$$

As $\beta \in W^{1,2}\left(0, T ; L^{2}\left(\Gamma_{3}\right)\right)$ we have for $\tau \in\left[t_{k}, t_{k+1}\right]$

$$
\left\|\beta(\tau)-\beta\left(t_{k+1}\right)\right\|_{L^{2}\left(\Gamma_{3}\right)} \leq \max _{\tau, t \in[0, T],|t-\tau| \leq \Delta t}\|\beta(\tau)-\beta(t)\|_{L^{2}\left(\Gamma_{3}\right)}=w(\beta, \Delta t) \text { with } \lim _{\Delta t \rightarrow 0} w(\beta, \Delta t)=0 .
$$

Therefore one obtains

$$
\left\|\epsilon_{n}^{k}\right\|_{L^{2}\left(\Gamma_{3}\right)} \leq D_{4}^{\prime \prime} \Delta t w(\beta, \Delta t)+\int_{t_{k}}^{t_{k+1}} D_{5}^{\prime \prime}\left\|\mathbf{u}^{2}(\tau)-\mathbf{u}^{2}\left(t_{k+1}\right)\right\|_{\left[L^{2}\left(\Gamma_{3}\right)\right]^{2}} \mathrm{~d} \tau .
$$

We deduce that

$$
\begin{aligned}
\left\|e_{n}^{i}\right\|_{L^{2}\left(\Gamma_{3}\right)} \leq \sum_{k=0}^{i-1} \exp \left[\frac{D_{2}^{\prime \prime}}{1-D_{2}^{\prime \prime} \Delta t}\left(t_{i}-t_{k+1}\right)\right]\left[D_{3}^{\prime \prime} \Delta t \| \mathbf{u}^{2}\left(t_{k+1}\right)-\right. & \left(\mathbf{u}^{k+1}\right)^{2} \|_{\left[L^{2}\left(\Gamma_{3}\right)\right]^{2}}+D_{4}^{\prime \prime} \Delta t w(\beta, \Delta t) \\
& \left.+\int_{t_{k}}^{t_{k+1}} D_{5}^{\prime \prime}\left\|\mathbf{u}^{2}(\tau)-\mathbf{u}^{2}\left(t_{k+1}\right)\right\|_{\left[L^{2}\left(\Gamma_{3}\right)\right]^{2}} \mathrm{~d} \tau\right] .
\end{aligned}
$$

We conclude that

$$
\begin{aligned}
\max _{i=0, . ., n}\left\|e_{n}^{i}\right\|_{L^{2}\left(\Gamma_{3}\right)} \leq \exp \left[\frac{D_{2}^{\prime \prime}}{1-D_{2}^{\prime \prime} \Delta t} T\right] \int_{0}^{T}\left[D_{3}^{\prime \prime}\left\|\hat{\mathbf{u}}_{n}^{2}(\tau)-\mathbf{u}_{n}^{2}(\tau)\right\|_{\left[L^{2}\left(\Gamma_{3}\right)\right]^{2}}\right. & D_{4}^{\prime \prime} w(\beta, \Delta t) \\
& \left.+D_{5}^{\prime \prime}\left\|\mathbf{u}^{2}(\tau)-\hat{\mathbf{u}}_{n}^{2}(\tau)\right\|_{\left[L^{2}\left(\Gamma_{3}\right)\right]^{2}}\right] \mathrm{d} \tau
\end{aligned}
$$

where $\left.\left.\hat{\mathbf{u}}_{n}(t)=\mathbf{u}\left(t_{k+1}\right), \forall t \in\right] t_{k}, t_{k+1}\right]$ and $\hat{\mathbf{u}}_{n}(0)=\mathbf{u}(0)$. To conclude we pass to the limit as $n \rightarrow+\infty$. 
Theorem 3.6. We have the following convergence: $\lim _{n \rightarrow+\infty} \max _{t \in[0, T]}\left\|\beta(t)-\beta_{n}(t)\right\|_{L^{2}\left(\Gamma_{3}\right)}=0$.

Proof. First we have

$$
\left\|\beta(t)-\beta_{n}(t)\right\|_{L^{2}\left(\Gamma_{3}\right)} \leq\left\|\beta(t)-\beta\left(t_{i+1}\right)\right\|_{L^{2}\left(\Gamma_{3}\right)}+\left\|e_{n}^{i+1}\right\|_{L^{2}\left(\Gamma_{3}\right)},
$$

so that

$$
\max _{t \in[0, T]}\left\|\beta(t)-\beta_{n}(t)\right\|_{L^{2}\left(\Gamma_{3}\right)} \leq \max _{s, t \in[0, T],|t-s|<\Delta t}\|\beta(t)-\beta(s)\|_{L^{2}\left(\Gamma_{3}\right)}+\max _{t \in[0, T]}\left\|e_{n}^{i+1}\right\|_{L^{2}\left(\Gamma_{3}\right)}
$$

and passing to the limit

$$
\lim _{n \rightarrow+\infty} \max _{t \in[0, T]}\left\|\beta(t)-\beta_{n}(t)\right\|_{L^{2}\left(\Gamma_{3}\right)} \leq \lim _{n \rightarrow+\infty} w\left(\beta, \frac{T}{n}\right)+\lim _{n \rightarrow+\infty} \max _{i=0, \ldots, n}\left\|e_{n}^{i}\right\|_{L^{2}\left(\Gamma_{3}\right)}=0 .
$$

Lemma 3.7. For all $\mathbf{v} \in L^{2}(0, T ; V)$ we have:

$$
\begin{aligned}
& \lim _{n \rightarrow+\infty} \int_{0}^{T}\left(\phi_{n}(t), \mathbf{v}(t)-\frac{\mathrm{d}}{\mathrm{d} t} \tilde{\mathbf{u}}_{n}(t)\right) \mathrm{d} t=\int_{0}^{T}(\phi(t), \mathbf{v}(t)-\dot{\mathbf{u}}(t)) \mathrm{d} t, \\
& \lim _{n \rightarrow+\infty} \int_{0}^{T}\left[\boldsymbol{\psi}_{n}(t), \mathbf{v}(t)-\frac{\mathrm{d}}{\mathrm{d} t} \tilde{\mathbf{u}}_{n}(t)\right]_{2} \mathrm{~d} t=\int_{0}^{T}[\boldsymbol{\psi}(t), \mathbf{v}(t)-\dot{\mathbf{u}}(t)]_{2} \mathrm{~d} t, \\
& \lim _{n \rightarrow+\infty} \int_{0}^{T} j\left(\beta_{n}, \mathbf{u}_{n}(t), \mathbf{v}(t)\right) \mathrm{d} t=\int_{0}^{T} j(\beta, \mathbf{u}(t), \mathbf{v}(t)) \mathrm{d} t, \\
& \lim _{n \rightarrow+\infty} \int_{0}^{T} a\left(\mathbf{u}_{n}(t), \mathbf{v}(t)\right) \mathrm{d} t=\int_{0}^{T} a(\mathbf{u}(t), \mathbf{v}(t)) \mathrm{d} t, \\
& \lim _{n \rightarrow+\infty} \int_{0}^{T} c\left(\beta_{n}(t), \mathbf{u}_{n}(t), \mathbf{v}(t)\right) \mathrm{d} t=\int_{0}^{T} c(\beta(t), \mathbf{u}(t), \mathbf{v}(t)) \mathrm{d} t .
\end{aligned}
$$

Proof. Inequality (52) and (53) are obtained by the strong convergence of $\left(\phi_{n}\right)_{n \in \mathbb{N}^{*}}$ and $\left(\boldsymbol{\psi}_{n}\right)_{n \in \mathbb{N}^{*}}$ towards $\phi$ and respectively $\boldsymbol{\psi}$. The weak convergence of $\left(\mathbf{u}_{n}(t)\right)_{n \in \mathbb{N}^{*}}$ towards $\mathbf{u}(t), \forall t \in[0, T]$ proves inequalities (54), (55) and (56).

Lemma 3.8. We have the following relations:

$$
\begin{aligned}
& \lim _{n \rightarrow+\infty} \inf \int_{0}^{T} j\left(\beta_{n}(t), \mathbf{u}_{n}(t), \frac{\mathrm{d}}{\mathrm{d} t} \tilde{\mathbf{u}}_{n}(t)\right) \mathrm{d} t \geq \int_{0}^{T} j(\beta(t), \mathbf{u}(t), \dot{\mathbf{u}}(t)) \mathrm{d} t, \\
& \lim _{n \rightarrow+\infty} \inf \int_{0}^{T} a\left(\mathbf{u}_{n}(t), \frac{\mathrm{d}}{\mathrm{d} t} \tilde{\mathbf{u}}_{n}(t)\right) \mathrm{d} t \geq \int_{0}^{T} a(\mathbf{u}(t), \dot{\mathbf{u}}(t)) \mathrm{d} t, \\
& \lim _{n \rightarrow+\infty} \int_{0}^{T} c\left(\beta_{n}(t), \mathbf{u}_{n}(t), \frac{\mathrm{d}}{\mathrm{d} t} \tilde{\mathbf{u}}_{n}(t)\right) \mathrm{d} t=\int_{0}^{T} c(\beta(t), \mathbf{u}(t), \dot{\mathbf{u}}(t)) \mathrm{d} t .
\end{aligned}
$$

Proof. First, we have

$$
\begin{aligned}
& \left|\int_{0}^{T}\left(j\left(\beta_{n}(t), \mathbf{u}_{n}(t), \frac{\mathrm{d}}{\mathrm{d} t} \tilde{\mathbf{u}}(t)\right)-j\left(\beta(t), \mathbf{u}(t), \frac{\mathrm{d}}{\mathrm{d} t} \tilde{\mathbf{u}}_{n}(t)\right)\right) \mathrm{d} t\right| \leq \\
& \qquad \int_{0}^{T}\left\|\varpi\left(\sigma_{N}\left(\mathbf{u}_{n}-\mathbf{u}\right)+C_{N}\left(\beta_{n}^{2} \mathbf{u}_{n N}-\beta^{2} \mathbf{u}_{N}\right)\right)\right\|_{H^{-\frac{1}{2}\left(\Gamma_{3}\right)}} \times\left\|\mu\left|\frac{\mathrm{d}}{\mathrm{d} t} \tilde{\mathbf{u}}_{n T}(t)\right|\right\|_{H^{\frac{1}{2}\left(\Gamma_{3}\right)}} \mathrm{d} t .
\end{aligned}
$$


As $\varpi\left(\sigma_{N}\left(\mathbf{u}_{n}\right)+C_{N} \beta_{n}^{2} u_{n N}\right)$ converges weakly towards $\varpi\left(\sigma_{N}(\mathbf{u})+C_{N} \beta^{2} u_{N}\right)$ in $H^{-\frac{1}{2}+\alpha}\left(\Gamma_{3}\right)$ for all $t$ we have

$$
\lim _{n \rightarrow+\infty}\left\|\varpi\left(\sigma_{N}\left(\mathbf{u}_{n}-\mathbf{u}\right)+C_{N}\left(\beta_{n}^{2} \mathbf{u}_{n}-\beta^{2} \mathbf{u}\right)\right)\right\|_{H^{-\frac{1}{2}\left(\Gamma_{3}\right)}}=0 .
$$

The result follows by Lebesgue's theorem and the fact that as in [8], we have

$$
\lim _{n \rightarrow+\infty} \inf \int_{0}^{T} j\left(\beta(t), \mathbf{u}(t), \frac{\mathrm{d}}{\mathrm{d} t} \tilde{\mathbf{u}}_{n}(t)\right) \mathrm{d} t \geq \int_{0}^{T} j(\beta(t), \mathbf{u}(t), \dot{\mathbf{u}}(t)) \mathrm{d} t .
$$

The proof of inequality (58) is similar to the one of [8].

By the compact imbedding of $H^{\frac{1}{2}}(\partial \Omega)$ in $L^{2}(\partial \Omega)$, we have that $\left(\mathbf{u}_{n}(t)\right)_{n \in \mathbb{N}^{*}}$ converges strongly towards $\mathbf{u}(t)$ in $\left[L^{2}\left(\Gamma_{3}\right)\right]^{2}$ for almost all t. The same convergence property holds for $\left(\beta_{n} \mathbf{u}_{n}\right)_{n \in \mathbb{N}^{*}}$, thus relation (59) follows.

Therefore the proof is complete.

Theorem 3.9. The elements $(\mathbf{u}, \beta)$ satisfy Problem $P_{2}$.

Proof. First, we show that the unilateral condition is satisfied. For all $t \in] 0, T]$, it follows from the definition of $\left(\mathbf{u}_{n}\right)_{n \in \mathbb{N}^{*}}$ and inequality (46) that

$$
\begin{aligned}
a\left(\mathbf{u}_{n}(t), \mathbf{v}(t)\right. & \left.-\mathbf{u}_{n}(t)\right)+c\left(\beta_{n}(t), \mathbf{u}_{n}(t), \mathbf{v}(t)-\mathbf{u}_{n}(t)\right)+j\left(\beta_{n}(t), \mathbf{u}_{n}(t), \mathbf{v}(t)-\mathbf{u}_{n}\left(t-\frac{T}{n}\right)\right) \\
& -j\left(\beta_{n}(t), \mathbf{u}_{n}(t), \mathbf{u}_{n}(t)-\mathbf{u}_{n}\left(t-\frac{T}{n}\right)\right) \geq\left(\phi_{n}, \mathbf{v}(t)-\mathbf{u}_{n}(t)\right)+\left[\boldsymbol{\psi}_{n}, \mathbf{v}(t)-\mathbf{u}_{n}(t)\right]_{2} \quad \forall \mathbf{v} \in K .
\end{aligned}
$$

We have also the following estimate for all $\boldsymbol{\vartheta} \in V$, by using (51)

$$
\begin{aligned}
\left(\mathbf{u}_{n}\left(t-\frac{T}{n}\right)-\mathbf{u}(t), \boldsymbol{\vartheta}\right) \leq & \left\|\mathbf{u}_{n}\left(t-\frac{T}{n}\right)-\mathbf{u}(t)\right\|_{\left[H^{1}(\Omega)\right]^{2}}\|\boldsymbol{\vartheta}\|_{\left[H^{1}(\Omega)\right]^{2}}+\left(\mathbf{u}_{n}(t)-\mathbf{u}(t), \boldsymbol{\vartheta}\right) \\
\leq & D_{5}\|\boldsymbol{\vartheta}\|_{\left[H^{1}(\Omega)\right]^{2}} \int_{t-\frac{T}{n}}^{\min \left(t+\frac{T}{n}, T\right)}\left(1+\|\dot{\boldsymbol{\phi}}(\tau)\|_{\left[L^{2}(\Omega)\right]^{2}}+\|\dot{\boldsymbol{\psi}}(\tau)\|_{\left[H^{-\frac{1}{2}}(\partial \Omega)\right]^{d}}\right) \mathrm{d} \tau \\
& +\left(\mathbf{u}_{n}(t)-\mathbf{u}(t), \boldsymbol{\vartheta}\right) .
\end{aligned}
$$

so we obtain that $\mathbf{u}_{n}\left(t-\frac{T}{n}\right)$ converges weakly towards $\mathbf{u}(t)$ in V. By passing to the limit in (60) we have

$$
a(\mathbf{u}(t), \mathbf{v}-\mathbf{u}(t))+c(\beta(t), \mathbf{u}(t), \mathbf{v}-\mathbf{u}(t))+j(\beta(t), \mathbf{u}(t), \mathbf{v}-\mathbf{u}(t)) \geq(\phi(t), \mathbf{v}-\mathbf{u}(t))+[\boldsymbol{\psi}, \mathbf{v}-\mathbf{u}(t)]_{2} \quad \forall \mathbf{v} \in K
$$

which implies by Green's formula that for all $t \in[0, T]$

$$
\left[\theta \sigma_{N}(\mathbf{u}(t)), v_{N}-u_{N}\right]+c_{N}(\beta(t), \mathbf{u}(t), \mathbf{v}(t)-\mathbf{u}(t)) \geq 0 \quad \forall \mathbf{v} \in K .
$$

Now, as $\left(\sigma_{N}\left(\mathbf{u}^{i+1}\right)+C_{N}\left(\beta^{i+1}\right)^{2} u_{N}^{i+1}\right) \in C^{*-}$ and $\mathbf{u}^{i} \in K$ we have:

$$
\left[\theta \sigma_{N}\left(\mathbf{u}^{i+1}\right), v_{N}^{\prime}-\frac{u_{N}^{i+1}-u_{N}^{i}}{\Delta t}\right]+c_{N}\left(\beta^{i+1}, \mathbf{u}^{i+1}, \mathbf{v}^{\prime}-\frac{\mathbf{u}^{i+1}-\mathbf{u}^{i}}{\Delta t}\right) \geq\left[\theta \sigma_{N}\left(\mathbf{u}^{i+1}\right), v_{N}^{\prime}\right]+c_{N}\left(\beta^{i+1}, \mathbf{u}^{i+1}, \mathbf{v}^{\prime}\right) .
$$

Therefore by setting $\mathbf{v}=\mathbf{v}^{\prime} \Delta t+\mathbf{u}^{i}$ in (6), we deduce for all $i=0, . ., n$, the following inequality:

$$
\begin{aligned}
& a\left(\mathbf{u}^{i+1}, \mathbf{v}^{\prime}-\frac{\Delta \mathbf{u}^{i}}{\Delta t}\right)+ c\left(\beta^{i+1}, \mathbf{u}^{i+1}, \mathbf{v}^{\prime}-\frac{\Delta \mathbf{u}^{i}}{\Delta t}\right)+j\left(\beta^{i+1}, \mathbf{u}^{i+1}, \mathbf{v}^{\prime}\right)-j\left(\beta^{i+1}, \mathbf{u}^{i+1}, \frac{\Delta \mathbf{u}^{i}}{\Delta t}\right) \geq \\
&\left(\phi^{i+1}, \mathbf{v}^{\prime}-\frac{\Delta \mathbf{u}^{i}}{\Delta t}\right)+\left[\boldsymbol{\psi}^{i+1}, \mathbf{v}^{\prime}-\frac{\Delta \mathbf{u}^{i}}{\Delta t}\right]_{2}+\left[\theta \sigma_{N}\left(\mathbf{u}^{i+1}\right), v_{N}^{\prime}\right]+c_{N}\left(\beta^{i+1}, \mathbf{u}^{i+1}, \mathbf{v}^{\prime}\right) \quad \forall \mathbf{v}^{\prime} \in V .
\end{aligned}
$$


By integrating the previous inequality with respect to time we obtain:

$$
\begin{aligned}
& \int_{0}^{T}\left\{\left(a\left(\mathbf{u}_{n}(t), \mathbf{v}^{\prime}(t)-\frac{\mathrm{d} \tilde{\mathbf{u}}_{n}}{\mathrm{~d} t}(t)\right)+c\left(\beta_{n}(t), \mathbf{u}_{n}(t), \mathbf{v}^{\prime}(t)-\frac{\mathrm{d} \tilde{\mathbf{u}}_{n}}{\mathrm{~d} t}(t)\right)+j\left(\beta_{n}(t), \mathbf{u}_{n}(t), \mathbf{v}^{\prime}(t)\right)\right.\right. \\
&-\left.j\left(\beta_{n}(t), \mathbf{u}_{n}(t), \frac{\mathrm{d} \tilde{\mathbf{u}}_{n}}{\mathrm{~d} t}(t)\right)\right\} \mathrm{d} t \geq \int_{0}^{T}\left(\phi_{n}(t), \mathbf{v}^{\prime}(t)-\frac{\mathrm{d} \tilde{\mathbf{u}}_{n}}{\mathrm{~d} t}(t)\right) \mathrm{d} t \\
&+\int_{0}^{T}\left[\boldsymbol{\psi}_{n}(t), \mathbf{v}^{\prime}(t)-\frac{\mathrm{d} \tilde{\mathbf{u}}_{n}}{\mathrm{~d} t}(t)\right]_{2} \mathrm{~d} t+\int_{0}^{T}\left\{\left[\theta \sigma_{N}\left(\mathbf{u}_{n}(t)\right), v_{N}^{\prime}\right]+c_{N}\left(\beta_{n}(t), \mathbf{u}_{n}(t), \mathbf{v}^{\prime}\right)\right\} \mathrm{d} t
\end{aligned}
$$

By passing to the limit in the previous inequality using Lemmas 3.7, 3.8 and the following relation obtained by the unilateral contact condition

$$
\left[\theta \sigma_{N}(\mathbf{u}(t)), \dot{u}_{N}(t)\right]+c_{N}(\beta(t), \mathbf{u}(t), \dot{\mathbf{u}}(t))=0
$$

we have

$$
\begin{aligned}
\int_{0}^{T}\left\{a \left(\mathbf{u}(t), \mathbf{v}^{\prime}(t)\right.\right. & -\dot{\mathbf{u}}(t))+c\left(\beta(t), \mathbf{u}(t), \mathbf{v}^{\prime}(t)-\dot{\mathbf{u}}(t)\right)+j\left(\beta(t), \mathbf{u}(t), \mathbf{v}^{\prime}(t)\right) \\
& -j(\beta(t), \mathbf{u}(t), \dot{\mathbf{u}}(t))\} \mathrm{d} t \geq \int_{0}^{T}\left(\phi(t), \mathbf{v}^{\prime}(t)-\dot{\mathbf{u}}(t)\right) \mathrm{d} t \\
& +\int_{0}^{T}\left[\boldsymbol{\psi}(t), \mathbf{v}^{\prime}(t)-\dot{\mathbf{u}}(t)\right]_{2} \mathrm{~d} t+\int_{0}^{T}\left\{\left[\theta \sigma_{N}(\mathbf{u}(t)), v_{N}^{\prime}-\dot{u}_{N}(t)\right]+c_{N}\left(\beta(t), \mathbf{u}(t), \mathbf{v}^{\prime}-\dot{\mathbf{u}}(t)\right)\right\} \mathrm{d} t
\end{aligned}
$$

If we set in the previous estimate $\mathbf{v}^{\prime} \in L^{2}(0, T ; V)$ defined by $\mathbf{v}^{\prime}(\tau)=\mathbf{w}$ for $\tau \in[t, t+h]$ and $\mathbf{v}(\tau)=\dot{\mathbf{u}}(\tau)$ otherwise, we obtain:

$$
\begin{aligned}
\frac{1}{h} \int_{t}^{t+h}\{a(\mathbf{u}(\tau), \mathbf{w}-\dot{\mathbf{u}}(\tau)) & +c(\beta(\tau), \mathbf{u}(\tau), \mathbf{w}-\dot{\mathbf{u}}(\tau))+j(\beta(\tau), \mathbf{u}(\tau), \mathbf{w}) \\
& -j(\beta(\tau), \mathbf{u}(\tau), \dot{\mathbf{u}}(\tau))\}) \mathrm{d} \tau \geq \frac{1}{h} \int_{t}^{t+h}(\phi(\tau), \mathbf{w}-\dot{\mathbf{u}}(\tau)) \mathrm{d} \tau \\
& +\frac{1}{h} \int_{t}^{t+h}[\psi(\tau), \mathbf{w}-\dot{\mathbf{u}}(\tau)]_{2} \mathrm{~d} \tau+\frac{1}{h} \int_{t}^{t+h}\left\{\left[\theta \sigma_{N}(\mathbf{u}(\tau)), \mathbf{w}-\dot{\mathbf{u}}_{N}(\tau)\right]+c_{N}(\beta(\tau), \mathbf{u}(\tau), \mathbf{w}-\dot{\mathbf{u}}(\tau))\right\} \mathrm{d} \tau
\end{aligned}
$$

Passing to the limit as $h \rightarrow 0^{+}$, we obtain by Lebesgue's theorem that inequality (2) is satisfied for almost all $t \in] 0, T[$.

\section{REFERENCES}

[1] L. Cangémi, Frottement et adhérence: modèle, traitement numérique et application à l'interface fibre matrice. PhD thesis, University of Aix-Marseille II (1997).

[2] M. Raous, L. Cangémi and M. Cocu, Un modèle couplant adhérence et frottement pour le contact unilatéral entre deux solides déformables. C. R. Acad. Sci., Paris, Série II b, t. 325 (1997) 503-509.

[3] M. Raous, L. Cangémi and M. Cocu, A consistent model coupling adhesion friction and unilateral contact. Comput. Methods Appl. Mech. Engrg. 177 (1999) 383-399.

[4] R. Rocca, Existence of a solution for a quasistatic problem of unilateral contact with local friction. C. R. Acad. Sci., Paris, Série I. 328 (1999) 1253-1258.

[5] R. Rocca and M. Cocu, Existence and approximation of a solution to quasistatic Signorini problem with local friction. Int. J. Engrg. (to appear). 
[6] R. Rocca, Analyse mathématique et numérique de problèmes quasi statique de contact unilatéral avec frottement local de Coulomb en élasticité, Ph.D. Thesis. Université d'Aix Marseille I (2000).

[7] Andersson L.-E, Existence results for quasistatic contact problems with Coulomb friction. Appl. Math. Optim. (to appear).

[8] M. Cocu, E. Pratt and M. Raous, Formulation and approximation of quasistatic frictional contact. Int. J. Engrg. Sci. 34 (1996) 783-798.

[9] J. Jarušek, Contact problems with bounded friction coercive case. Czechoslovak Math. J. 33 (1983) 237-261.

[10] C. Eck and J. Jarušek, Existence results for the static contact problem with Coulomb friction, Math. Models Methods Appl. Sci. 8(1998) 445-468.

[11] R.A. Adams, Sobolev spaces. Academic Press, New York (1975).

[12] J.L. Lions and E. Magenes, Problèmes aux limites non homogènes, Vol. I. Dunod, Paris (1967).

[13] E. Zeidler, Nonlinear Functional Analysis and its Applications, Vol. I. Springer Verlag, New York (1993).

To access this journal online:

www.edpsciences.org 